\title{
Dementia Enlightened?! A Systematic Literature Review of the Influence of Indoor Environmental Light on the Health of Older Persons with Dementia in Long-Term Care Facilities
}

\author{
Ingrid Goudriaan (D) ${ }^{1,2}$ \\ Leonieke C van Boekel (D) \\ Marjolein EA Verbiest (D) \\ Joost van Hoof $\left(\mathbb{D}^{3,4}\right.$ \\ Katrien G Luijkx (D) \\ 'Tranzo, Tilburg School of Social and \\ Behavioral Sciences, Tilburg University, \\ Tilburg, the Netherlands; ${ }^{2}$ Innovation and \\ Quality, BrabantZorg, Oss, the \\ Netherlands; ${ }^{3}$ Chair of Urban Ageing, \\ Faculty of Social Work \& Education, The \\ Hague University of Applied Sciences, \\ The Hague, the Netherlands; ${ }^{4}$ Institute of \\ Spatial Management, Faculty of \\ Environmental Engineering and Geodesy, \\ Wrocław University of Environmental \\ and Life Sciences, Wrocław, Poland
}

\begin{abstract}
Light therapy for older persons with dementia is often administered with light boxes, even though indoor ambient light may more comfortably support the diverse lighting needs of this population. Our objective is to investigate the influence of indoor daylight and lighting on the health of older adults with dementia living in long-term care facilities. A systematic literature search was performed within PubMed, CINAHL, PsycINFO, Web of Science and Scopus databases. The included articles $(n=37)$ were published from 1991 to 2020. These articles researched the influence of existing and changed indoor light conditions on health and resulted in seven categories of health outcomes. Although no conclusive evidence was found to support the ability of indoor light to decrease challenging behaviors or improve circadian rhythms, findings of two studies indicate that exposure to (very) cool light of moderate intensity diminished agitation. Promising effects of indoor light were to reduce depressive symptoms and facilitate spatial orientation. Furthermore, there were indications that indoor light improved one's quality of life. Despite interventions with dynamic lighting having yielded little evidence of its efficacy, its potential has been insufficiently researched among this study population. This review provides a clear and comprehensive description of the impact of diverse indoor light conditions on the health of older adults with dementia living in long-term care facilities. Variation was seen in terms of research methods, (the description of) light conditions, and participants' characteristics (types and severity of dementia), thus confounding the reliability of the findings. The authors recommend further research to corroborate the beneficial effects of indoor light on depression and to clarify its role in supporting everyday activities of this population. An implication for practice in long-term care facilities is raising the awareness of the increased lighting needs of aged residents.
\end{abstract}

Keywords: lighting, Alzheimer's disease, assisted living, nursing homes, indoor daylight, light therapy

\section{Introduction}

Although Western governmental policies encourage aging-in-place, the number of persons with dementia living in long-term care facilities is still growing. According to the World Health Organization, ${ }^{1}$ dementia is one of the major causes of disability and dependency among older people. Therefore, we would expect long-term care facilities to offer an optimal physical environment that would support older persons
Correspondence: Ingrid Goudriaan Tranzo, Tilburg University, PO Box 90153 , Tilburg, 5000 LE, the Netherlands Tel +3I (0) 612692435

Email ingrid.goudriaan@xs4all.nl 
with dementia and accommodate for their losses. However, the physical environment, including indoor light, is often an undervalued and even ignored resource in dementia care. $^{2-4}$ Indoor daylight and lighting are essential elements of an optimal environment to compensate for age- and dementia-related sensory changes. ${ }^{5,6}$

Due to the aging of the eyes, older people have an increasing demand for higher light levels that support good vision and help synchronize their biological clock. $^{7,8}$ Sufficient light for visual needs helps older people to (independently) execute activities of daily living, hobbies and social activities. The light aids them in not only moving safely but to also feeling safe. ${ }^{9}$ In addition, high-intensity light during daytime is needed, because it is the strongest cue for synchronizing the biological clock with the 24-hour rhythm of the earth. ${ }^{10,11}$ In turn, the biological clock plays an important role in the timing and coordination of physiological and psychological processes with a circadian (24 h) rhythm, including hormone levels, body temperature, alertness, urine production and composition, sleepwake rhythm, mood and performance. ${ }^{12-15}$ Despite the lighting needs of older persons, the literature shows that the light conditions in long-term care facilities are poor, both for visual needs as well as for entraining the biological clock. ${ }^{16-19}$

Ensuring good quantity and quality of indoor light is even more important for older persons with dementia. For instance, in winter, this group is more sensitive to circadian disruption than healthy older adults. ${ }^{20}$ Increasing dementia severity can lead to increasing sleep-wake rhythm disturbances, which in extreme cases may lead to complete day and night sleep pattern reversals. ${ }^{21,22}$ In addition, specific dementia-related changes in the brain result in difficulties in finding objects, reading, depth perception, perceiving structure from motion, color recognition and impairment in spatial contrast sensitivity. ${ }^{23}$ It is to be expected that sufficient light that supports good vision can help compensate for these changes as well as improve orientation of older adults with dementia.

Light therapy, which focuses on changes in the circadian pacemaker in the brain, is an emerging therapy within the domain of dementia care. ${ }^{24}$ While light therapy can be administered in a number of ways, the use of a light box standing on a table in front of the person with dementia is to date the most frequently applied and researched method. ${ }^{25}$ However, using light boxes for persons with dementia presents some disadvantages. To remain sitting in front of a light box for a minimum of 30 minutes and up to 2 hours per day may be difficult for persons with dementia, ${ }^{26}$ even if the intervention takes place while performing other activities, such as having meals or watching television. The exposure may require supervision, ${ }^{26,27}$ which in turn puts a strain on the participants as well as on the supervisor. In contrast, indoor environmental light may be a preferable source because it allows for free movement. ${ }^{28}$ In recent years, the use of dynamic lighting for this population has become increasingly popular, not in the least due to the often unproven claims of their suppliers. Dynamic lighting changes during the day in illuminance or spectral composition, or both, in such a way that the variation in light can be

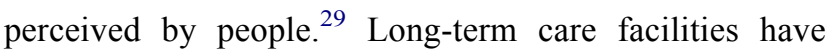
purchased dynamic lighting with the intention of improving the well-being and day-night rhythm of persons with dementia. $^{30}$

The foregoing paragraphs highlight the importance of indoor daylight and lighting and raises questions about the influence thereof. To date, there is no systematic review published that exclusively documents the influence of indoor environmental light conditions (daylight and lighting) on the health of older persons with dementia living in a long-term care facility. A Cochrane review on the effects of light therapy on persons with dementia included only one study with indoor environmental light. ${ }^{31}$ Their conclusions were based on studies where the light sources (light boxes, light visors, light fixtures) differed to such an extent that the results might not be comparable. Other reviews did not fully combine all three distinctive elements that this research is interested in: older adults with dementia, indoor environmental light and long-term care facilities. These reviews, for instance, were concerned with light therapy and dementia, ${ }^{32}$ daylight and health in general $^{33}$ or a therapeutic lighting design and older adults. $^{34}$ Therefore, the research question for this systematic literature review is the following: What does scientific literature tell us about the influence of indoor environmental light conditions (daylight and lighting) on the health of older adults with dementia living in a long-term care facility? Health is thereby defined as the ability to adapt and self-manage in the face of social, physical and emotional challenges. ${ }^{35}$ Compared to the WHO-definition of health, ${ }^{36}$ this definition of Huber et al better reflects the everyday reality of people with chronic diseases, including people with dementia. 


\section{Methods}

\section{Search Strategy}

To identify relevant empirical studies, we conducted a systematic literature search in five scientific databases. We focused on databases related to health, medicine, nursing, behavioral and social sciences, as well as the built environment. Therefore, the databases PubMed, CINAHL, PsycINFO, Web of Science and Scopus were selected. Published studies were next identified using a search strategy based on the three facets of the research question: persons with dementia, light and long-term care facilities (Supplementary Table). The search string encompassed specific indexing terms and subject headings from the different databases as well as keywords, synonyms and some additional words that best represented the facet. The search included articles published up to May 2020 and resulted in 810 unique articles.

\section{Article Selection}

Figure 1 shows the selection process of the articles. In the first selection phase, all duplicates were removed, and the remaining titles $(n=810)$ were screened for inclusion by the first author (IG). When all selection criteria (Table 1) were met or in any cases of doubt, articles proceeded for further screening. In this second phase, two reviewers (IG and MV) independently assessed the abstracts of the remaining articles $(n=354)$ and discussed the eligibility until they reached consensus. If consensus was not reached, the full research team was consulted. In the third phase, an identical procedure was followed for assessing the remaining full-text articles $(\mathrm{n}=99)$. Finally, the first author (IG) screened all references of the 35 included articles for any additional potentially relevant articles (snowball method). Eligibility of these articles was again checked by two reviewers independently (IG and LVB) and yielded two additional articles, which resulted in a total of 37 articles in this review.

\section{Data Processing}

Data from the 37 included articles were extracted independently by the first author and one of the other reviewers, and subsequently discussed in the same pairs (IG and $\mathrm{LVB} / \mathrm{MV} / \mathrm{JVH})$. All reviewers used the same data extraction form consisting of the following categories:

1. Study, sample and setting characteristics,
2. Specifics on the light condition, lighting systems, light measurements and light-measuring equipment (based on recommendations by Aarts, Aries, Diakoumis, van Hoof), ${ }^{38}$

3. Environmental properties (eg, location, date, weather conditions, daylight openings),

4. Health outcomes and assessment tools used,

5. Specifics on data collection methods and (statistical) analyses,

6. Appraisal of the methodological quality.

Because we included empirical studies regardless of their research design, we used the Mixed Methods Appraisal Tool (MMAT) for appraising a study's methodological quality. ${ }^{39}$ The MMAT was comprised of two general screening questions and five specific methodological criteria for each type of research design. The maximum score of each type of design was five.

\section{Results}

\section{Characteristics of Included Articles}

The majority of the studies were conducted in North America (51.4\%), followed by Europe (32.4\%), Asia (10.8\%), Australasia (2.7\%) and in both Asia and Australasia (2.7\%). For more detailed information about the characteristics and the results of the included articles see. The articles were published from 1991 to 2020 and written in English. Most studies took place in (dementiaspecific care units of) nursing homes, assisted living facilities or both. The research designs included the following categories: 12 quantitative descriptive studies, 19 quantitative non-randomized studies, 2 quantitative randomized controlled trials, 3 qualitative studies and 1 mixed methods study. Although the methodological quality of the studies varied, most studies reached MMAT quality scores of three or more out of five. Less attention was given to the results of two studies that received lower MMAT scores. $^{30,40}$ The light conditions were often insufficiently described; eg, a number of studies lacked relevant data, such as characteristics of the light sources, or used subjective light measurements.

Almost $60 \%(n=21)$ of the studies investigated the influence of existing indoor light conditions on health. In 16 studies (43.2\%), the indoor light conditions were purposely changed before or during the study. These light interventions during the day consisted of an increase in the light intensity, color temperature or 


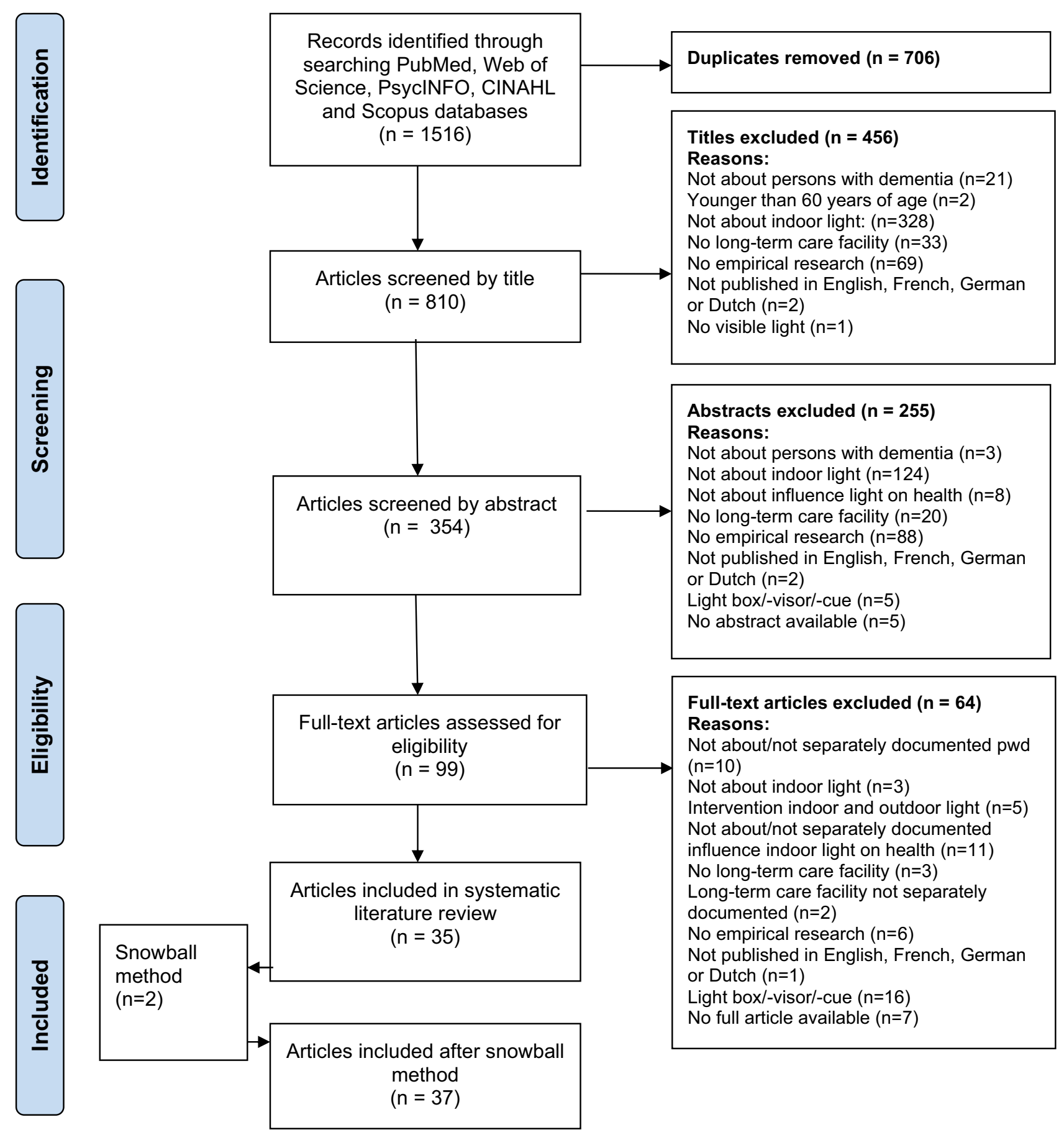

Figure I PRISMA flowchart of the literature search on the influence of indoor environmental light on the health of older persons with dementia in long-term care facilities. Adapted from Moher D, Liberati A, Tetzlaff J, Altman DG, The PRISMA Group (2009). Preferred Reporting Items for Systematic Reviews and Meta-Analyses: The PRISMA Statement. PLoS Med. 6(7):e I000097. ${ }^{37}$

both. This was done either by adding luminaires or replacing light bulbs, adding static or dynamic lighting systems, increasing the use of incident daylight through newly built skylights or by taking residents to a daylit room near the windows for socialization. Two interventions took place around night-time, namely reduction of the frequency of light changes during incontinence care and a naturalistic simulation of dawn-dusk signals in the bedrooms of persons with dementia. Most of the light interventions took place in communal living 
Table I Inclusion and Exclusion Criteria

\begin{tabular}{|l|l|}
\hline Inclusion Criteria & Exclusion Criteria \\
\hline $\begin{array}{l}\text { Research investigating the } \\
\text { influence of indoor daylight and/ } \\
\text { or lighting on health }\end{array}$ & $\begin{array}{l}\text { Research on interventions with } \\
\text { (supplementary) light boxes, light } \\
\text { visors, light cues or light tables }\end{array}$ \\
\hline $\begin{array}{l}\text { Research in which participants } \\
\text { are } 60 \text { years and older and have } \\
\text { dementia }\end{array}$ & $\begin{array}{l}\text { Research on immune } \\
\text { suppression by light }\end{array}$ \\
\hline $\begin{array}{l}\text { Research in which participants } \\
\text { live in a long-term care facility }\end{array}$ & $\begin{array}{l}\text { Research aimed at extra visual } \\
\text { effects of light on health (eg, } \\
\text { through the skin instead of the } \\
\text { eye) and/or on effects of non- } \\
\text { visible light }\end{array}$ \\
\hline $\begin{array}{l}\text { Empirical research with } \\
\text { a qualitative, quantitative or } \\
\text { mixed methods design }\end{array}$ & $\begin{array}{l}\text { Research conducted exclusively } \\
\text { in (geriatric) hospitals, long-term } \\
\text { care facilities for persons with } \\
\text { psychiatric problems or for } \\
\text { persons with intellectual } \\
\text { disabilities who also have } \\
\text { dementia }\end{array}$ \\
\hline
\end{tabular}

rooms, dining rooms, or both $(n=12)$ and the rest occurred in bedrooms $(n=3)$ or a small light therapy living room $(n=1)$.

\section{Influence of Light on Health}

By examining and reporting the influence of light on health, two specific characteristics of light were classified: light level and color temperature. Light can differ in intensity, the light level, and its spectrum can, for example, be experienced as warm white or yellowish, cool white or even bluish, often referred to as the color temperature. The correct technical term is correlated color temperature (CCT) and depends on the type of light source. In this review, these two characteristics are used separately or in different combinations to indicate the light level and (correlated) color temperature of light (Table 3).

By categorizing all health outcomes in the literature into thematic groups, we identified seven categories of health outcomes (number of articles; percentage). We will discuss the outcomes in the following order:

1. Behavior, such as agitation, (neuro)psychiatric symptoms and apathy $(\mathrm{n}=22 ; 59.5 \%)$
2. Daily functioning, such as activities of daily living, falls and food intake $(n=9 ; 24.3 \%)$

3. Mental functions, such as orientation, cognition and disturbances of consciousness $(n=7 ; 18.9 \%)$

4. Mood and emotions, such as depression and subjective emotions $(n=8 ; 21.6 \%)$

5. Quality of life $(n=4 ; 10.8 \%)$

6. (24h) Rest-activity rhythms or certain aspects thereof, such as daytime activity, rest-activity cycles, nocturnal restlessness and sleep $(n=18$; 48.6\%)

7. Bodily functions: adverse effects of light, use of medication and visual performance $(n=3 ; 8.1 \%)$.

\section{Behavior}

Behavior was the most investigated health outcome $(n=22)$ in the literature, and most articles reported on sets of challenging behaviors $(n=18 ; 81.8 \%)$, like agitation. The Cohen-Mansfield Agitation Inventory (CMAI), which consists of 29 distinctive behaviors, was often used in the research. ${ }^{41}$ Almost $30 \%(n=6)$ of the articles (also) researched one or more separate challenging behaviors, such as pacing or apathy. Only two studies also involved positive behaviors. In this review two types of influences of light on behavior were distinguished. First, articles were identified that investigated a direct influence of the quantity, quality or color of light on behavior, which we will discuss first $(n=21)$. Second, a more indirect influence of light on behavior was found, namely temporal aspects of light, like day length or time of day $(n=7)$.

\section{Direct Influence of Light on Behavior}

Six methodologically sound light intervention studies investigated the influence of cooler light (neutral to very cool) during the day on sets of challenging behaviors (MMAT score $\geq 3 / 5$ ). The majority of these light interventions $(n=4 ; 66.7 \%)$ did not yield any significant influences on sets of challenging behaviors in the intervention group compared to a control condition. ${ }^{27,42-44}$ These ineffective interventions concerned light with a (very) high light intensity (Table 3) which was produced by lighting installations or was a result of incident daylight through a window. In two other studies, the lighting interventions resulted in a daytime light exposure of moderate intensity of (very) cool light. ${ }^{45,46}$ In these before-after studies $\left(\mathrm{n}=14 ;^{45} \mathrm{n}=12^{46}\right)$, agitation decreased significantly compared to the baseline condition. One of these studies found that the decrease in agitation was attributable to 


\begin{tabular}{|c|c|c|c|}
\hline 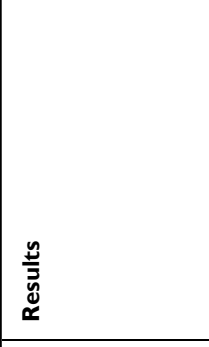 & 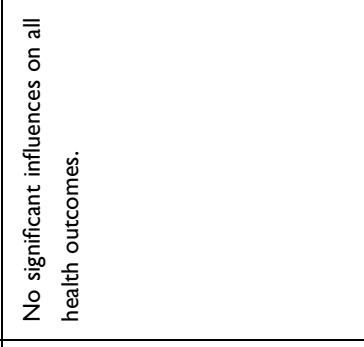 & 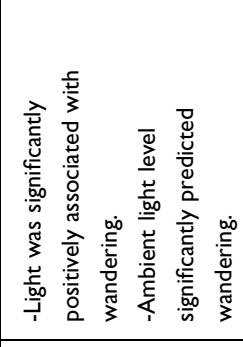 & 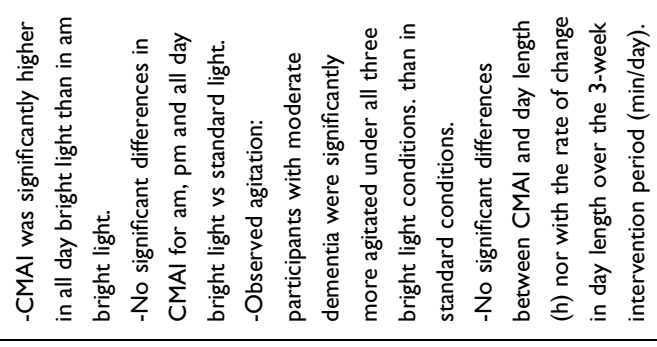 \\
\hline 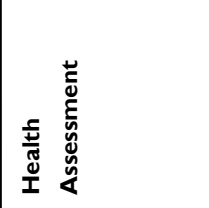 & 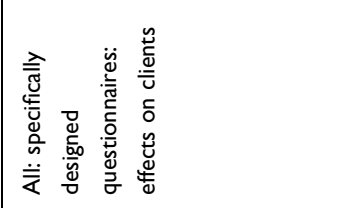 & 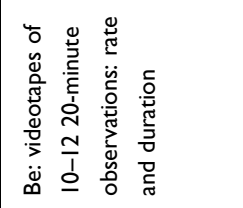 & 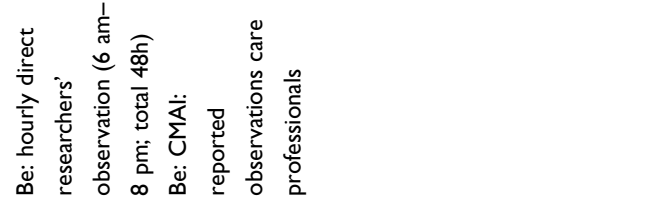 \\
\hline 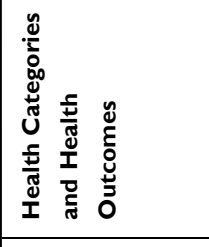 & 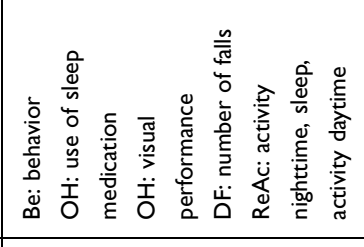 & 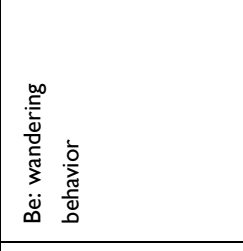 & 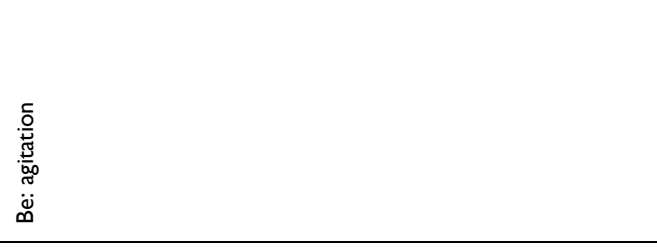 \\
\hline 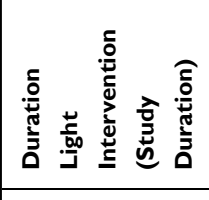 & 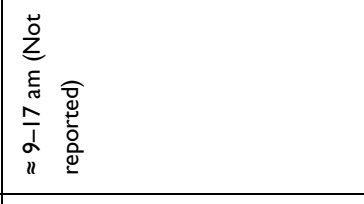 & 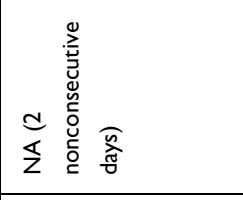 & 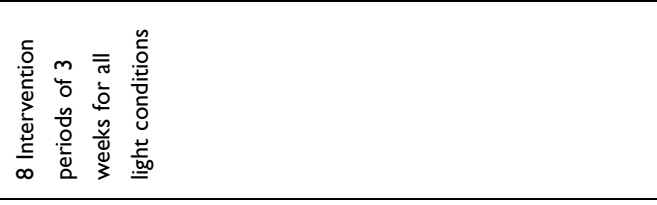 \\
\hline 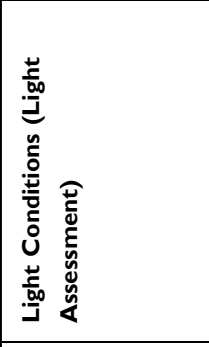 & 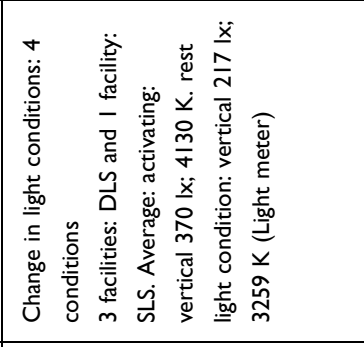 & 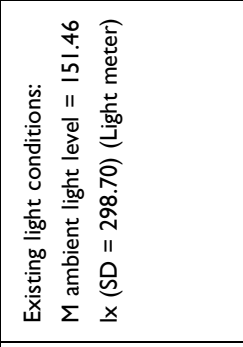 & 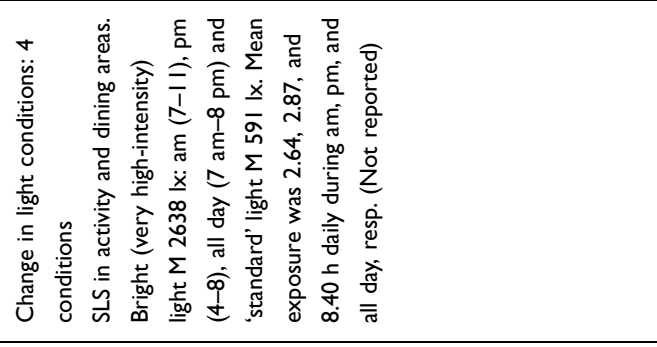 \\
\hline 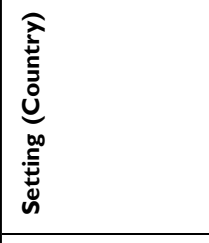 & 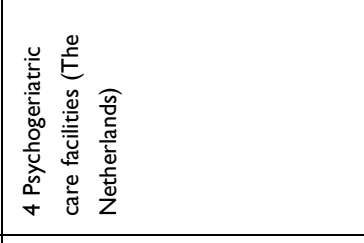 & 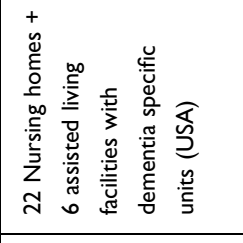 & 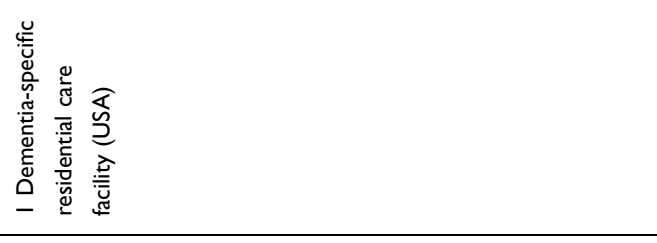 \\
\hline & 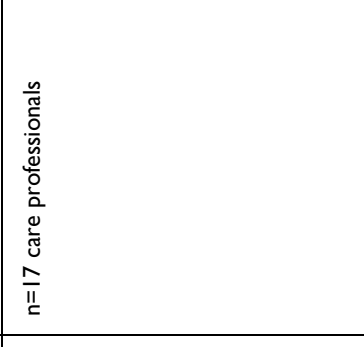 & 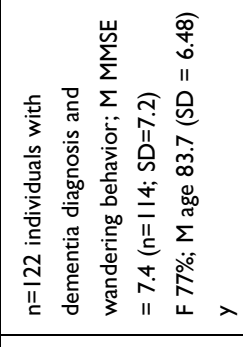 & 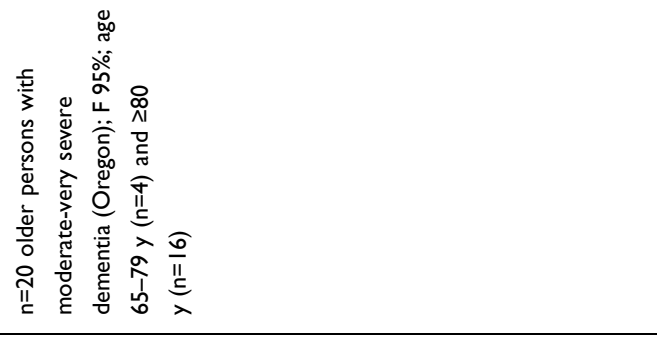 \\
\hline 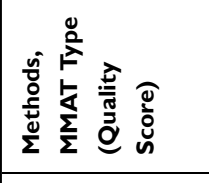 & 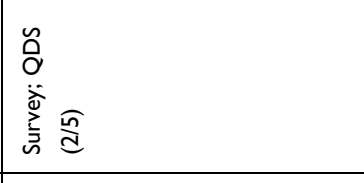 & 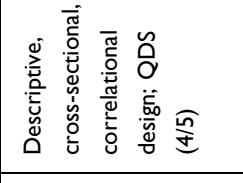 & 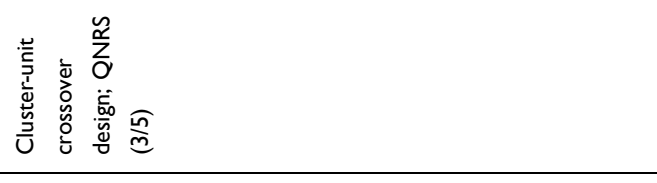 \\
\hline 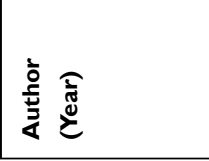 & 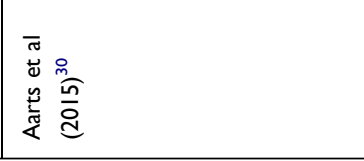 & 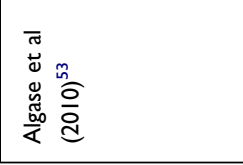 & 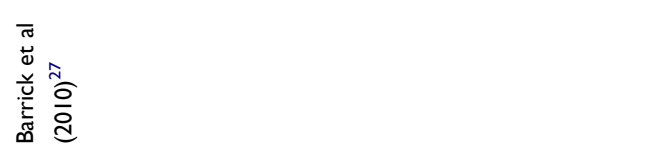 \\
\hline
\end{tabular}




\begin{tabular}{|c|c|c|}
\hline 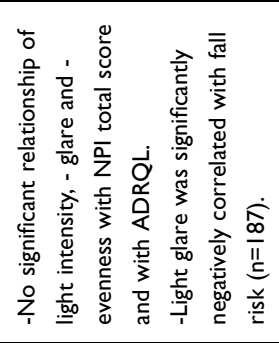 & 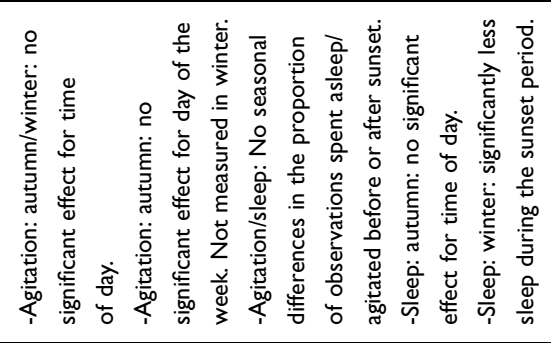 & 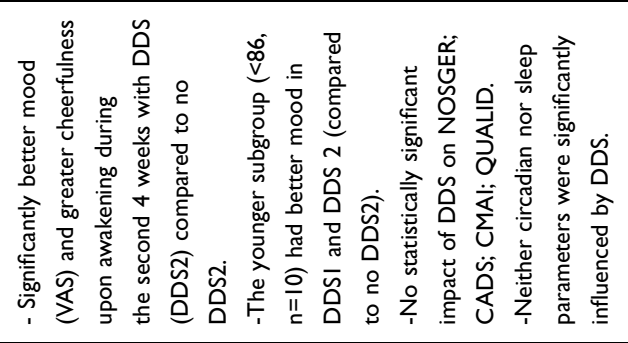 \\
\hline 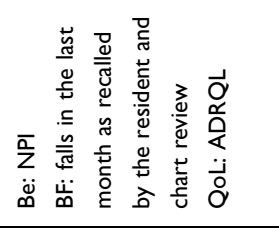 & 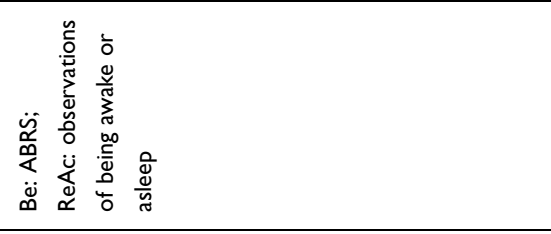 & 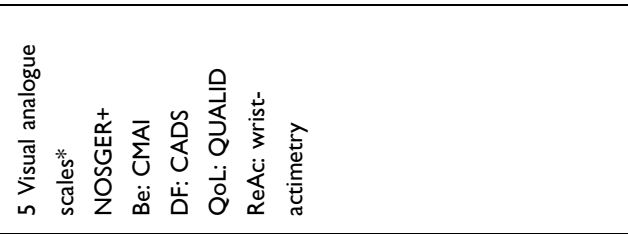 \\
\hline 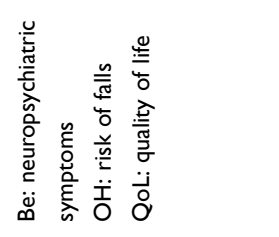 & 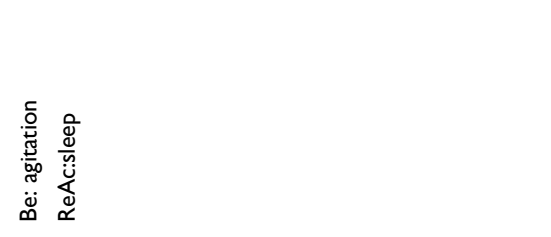 & 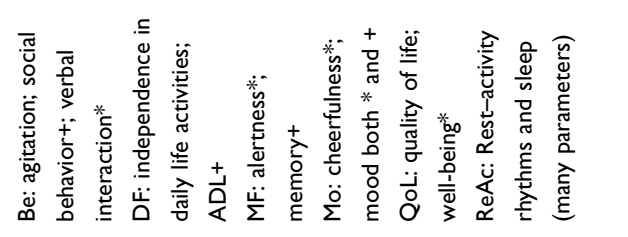 \\
\hline 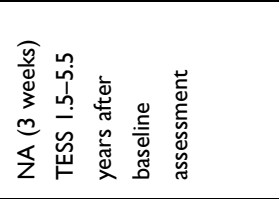 & 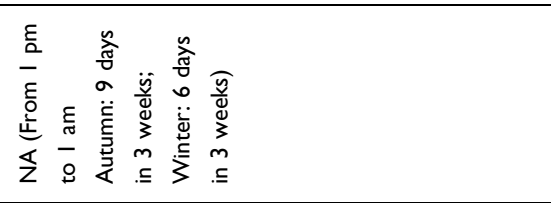 & 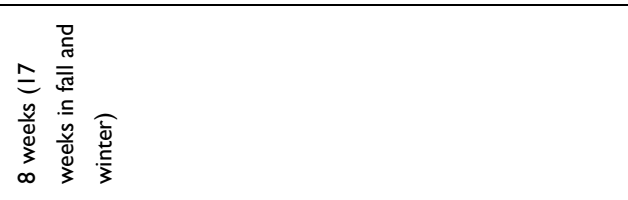 \\
\hline 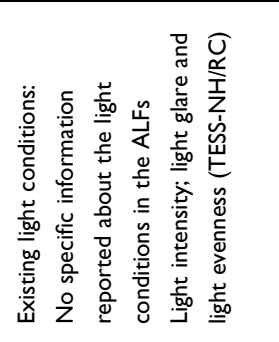 & 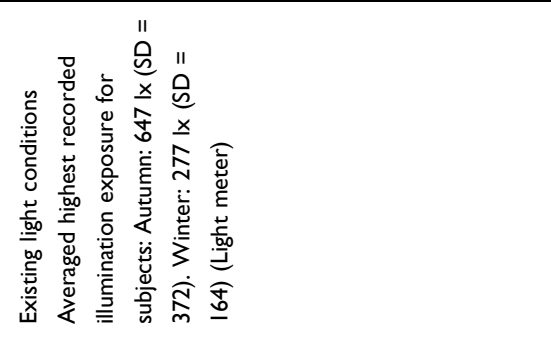 & 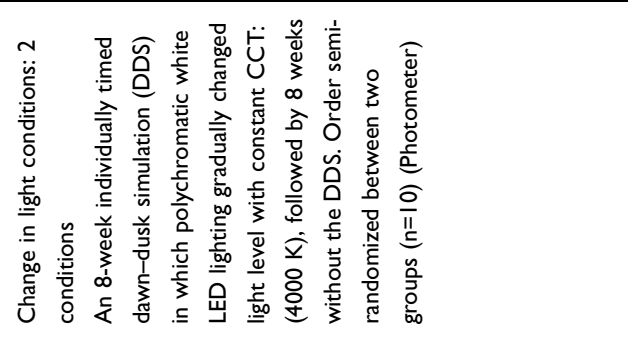 \\
\hline 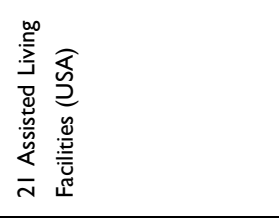 & 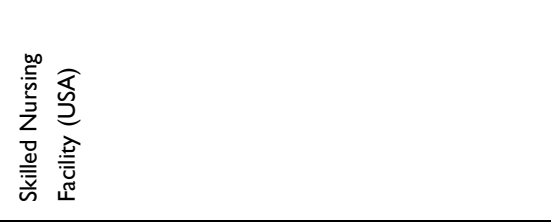 & 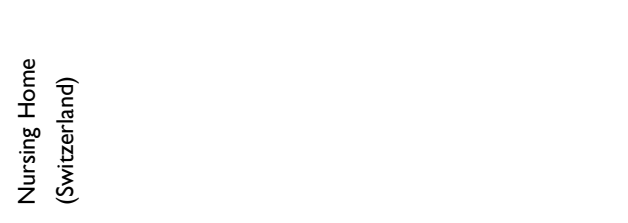 \\
\hline 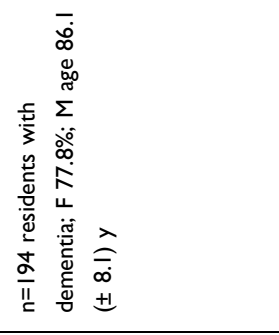 & 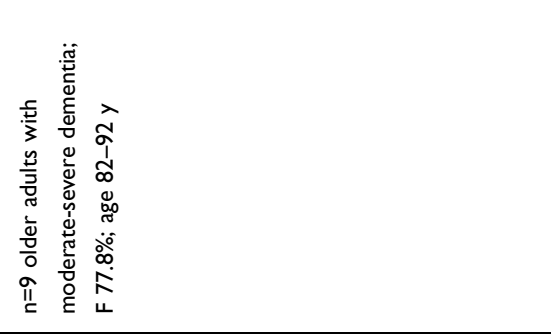 & 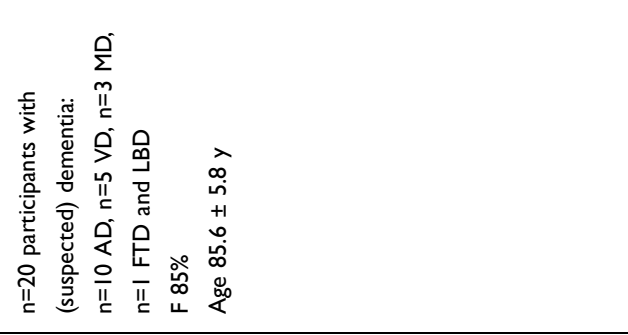 \\
\hline 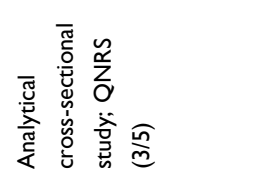 & 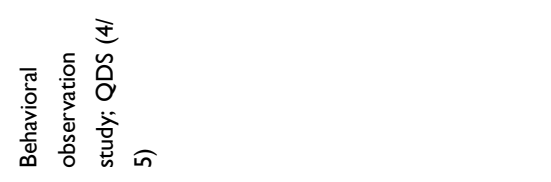 & 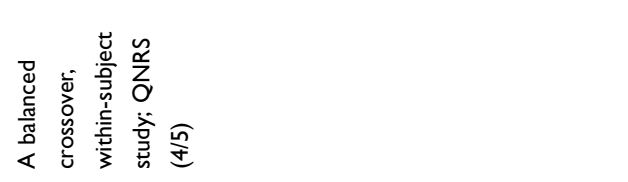 \\
\hline 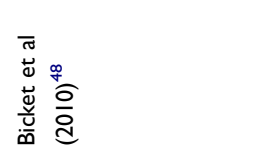 & 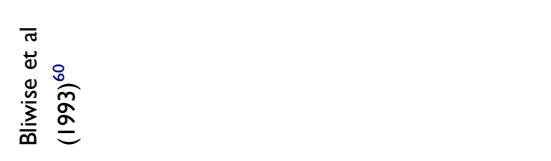 & 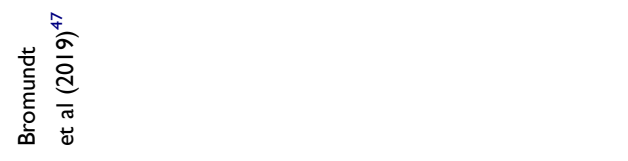 \\
\hline
\end{tabular}




\begin{tabular}{|c|c|c|c|c|}
\hline $\begin{array}{l}\frac{\underline{\underline{\nu}}}{\bar{z}} \\
\underline{x}\end{array}$ & 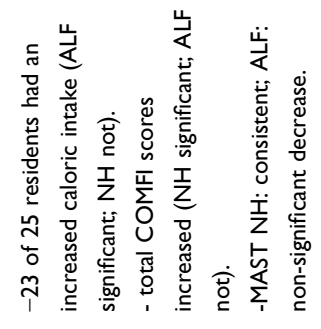 & 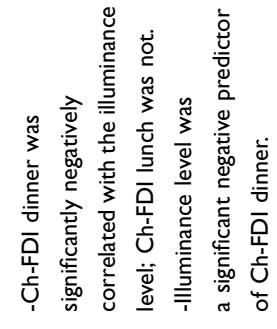 & 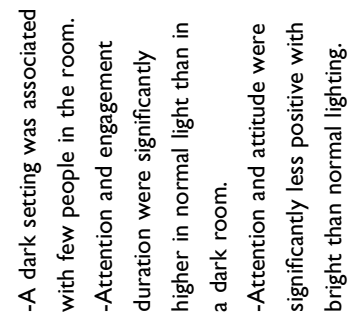 & 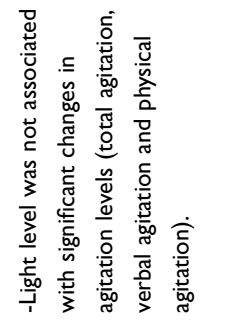 \\
\hline 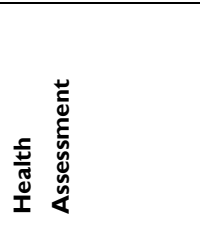 & 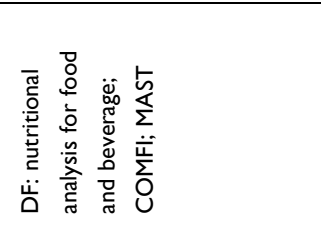 & 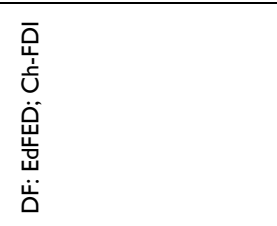 & 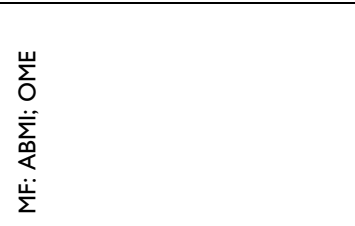 & 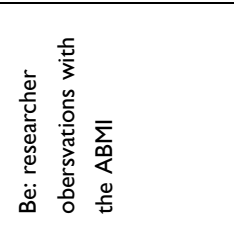 \\
\hline 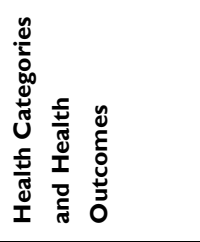 & 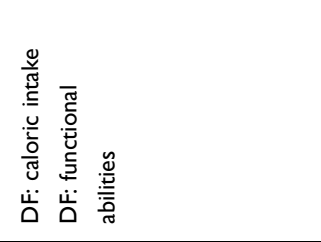 & 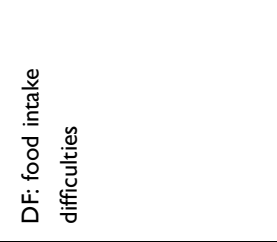 & 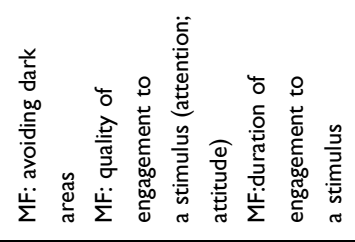 & 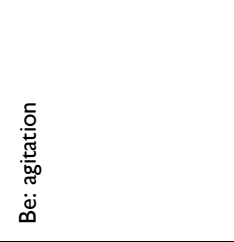 \\
\hline 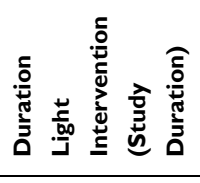 & 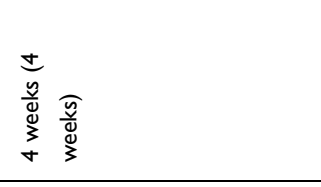 & 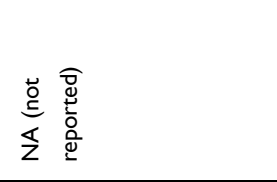 & $\begin{array}{l}\frac{\widehat{g}}{4} \\
\frac{1}{2} \\
\frac{m}{2} \\
\frac{n}{2} \\
\end{array}$ & 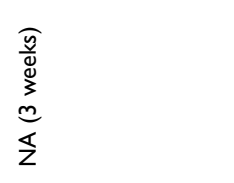 \\
\hline 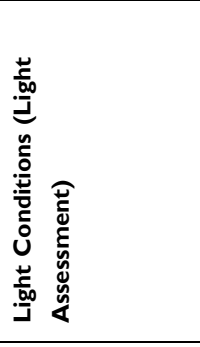 & 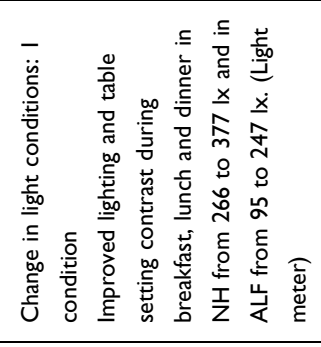 & 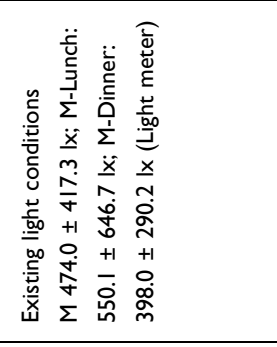 & 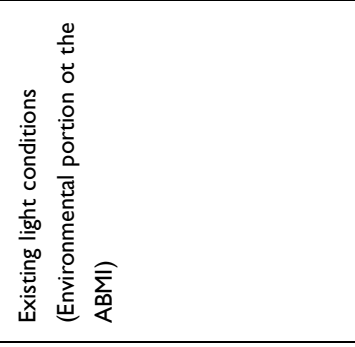 & 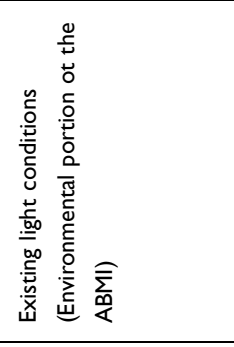 \\
\hline 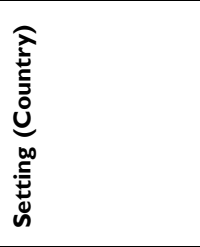 & 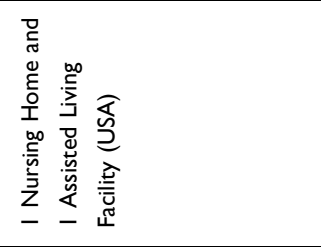 & 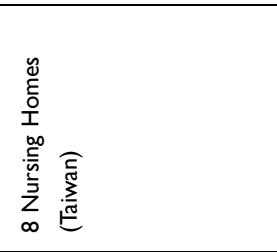 & 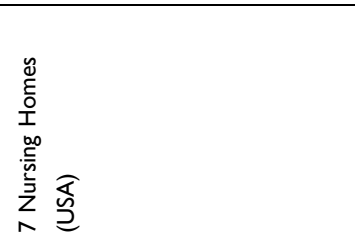 & 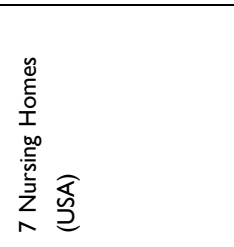 \\
\hline & 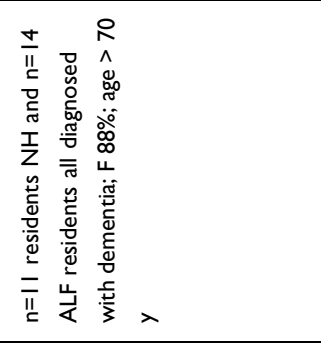 & 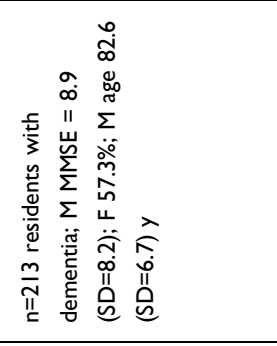 & 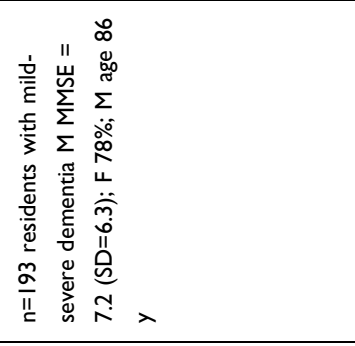 & 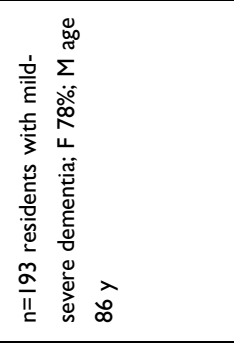 \\
\hline $\begin{array}{ll}0 \\
0\end{array}$ & 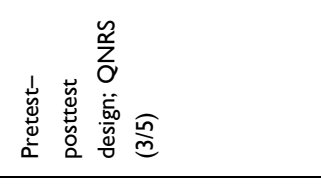 & 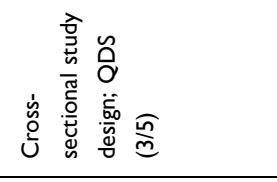 & 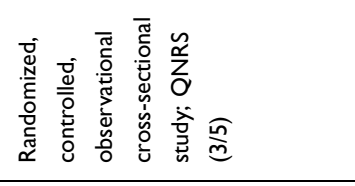 & 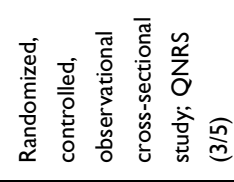 \\
\hline 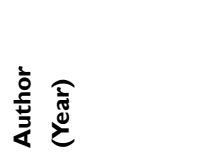 & 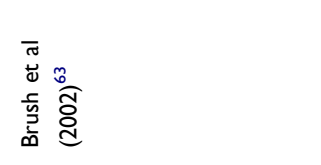 & 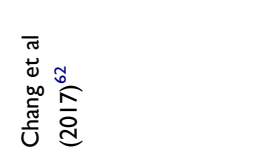 & 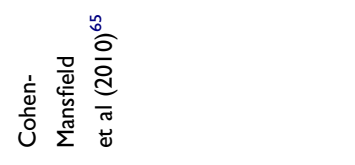 & 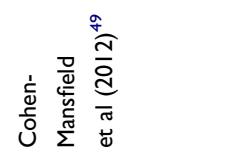 \\
\hline
\end{tabular}




\begin{tabular}{|c|c|c|}
\hline 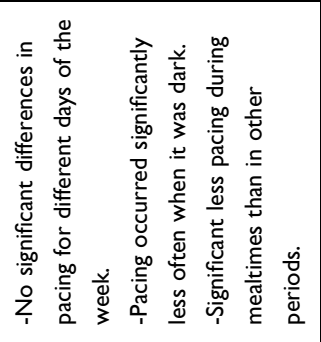 & 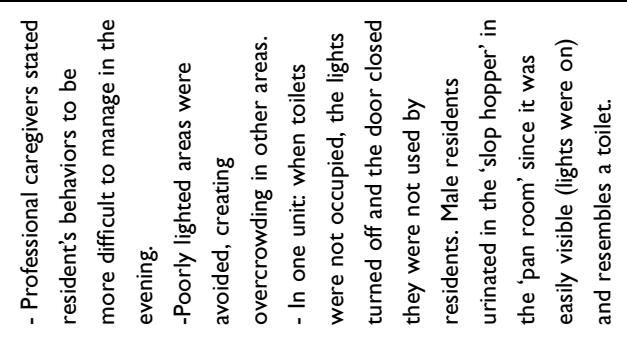 & 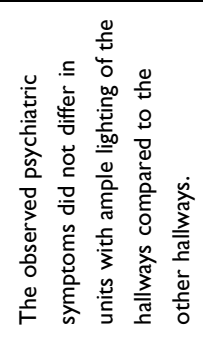 \\
\hline 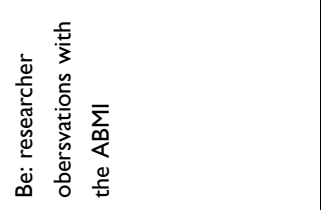 & 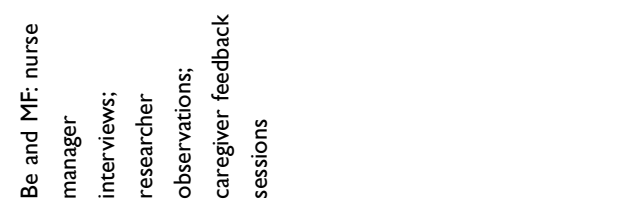 & 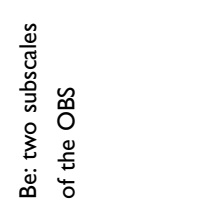 \\
\hline 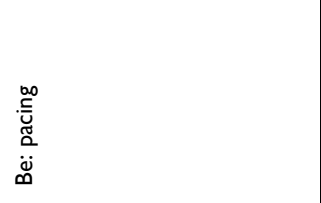 & 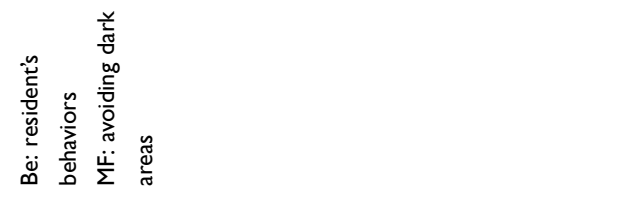 & 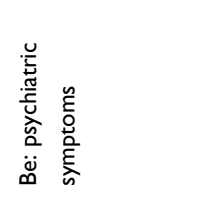 \\
\hline 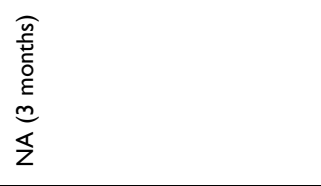 & 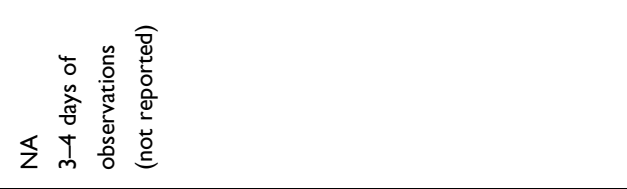 & 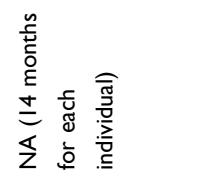 \\
\hline 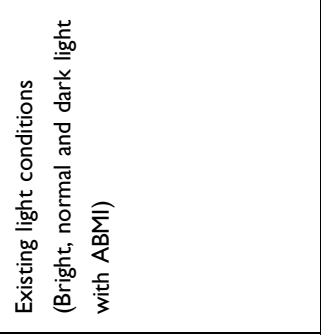 & 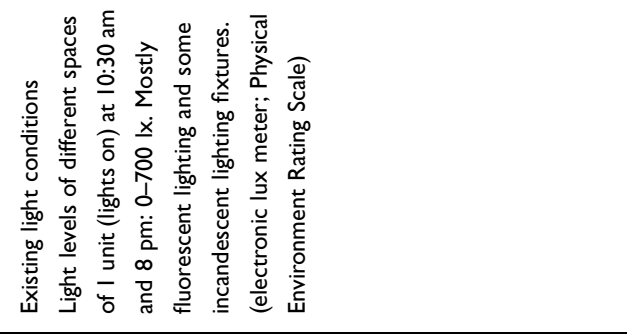 & 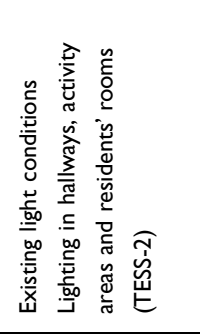 \\
\hline 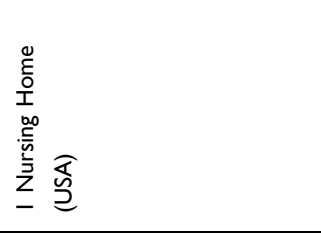 & 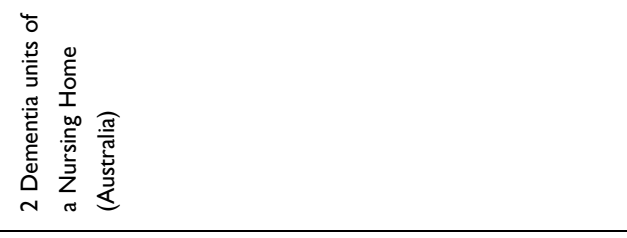 & 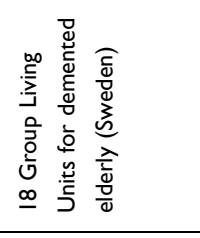 \\
\hline 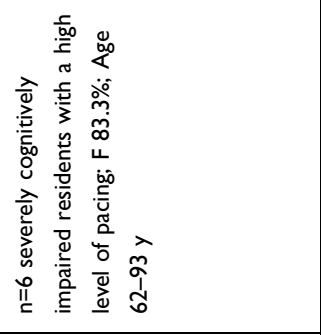 & 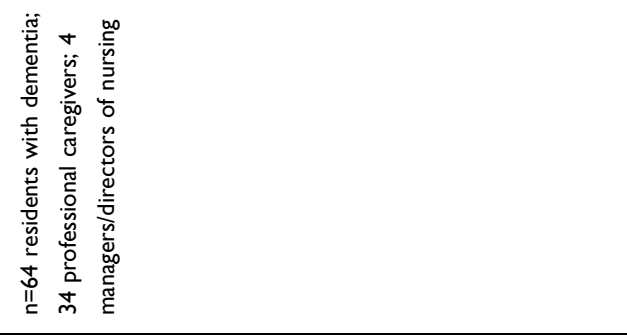 & 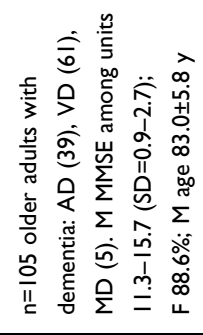 \\
\hline 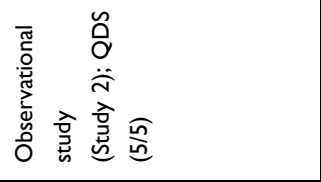 & 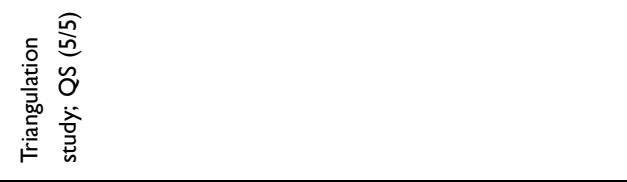 & 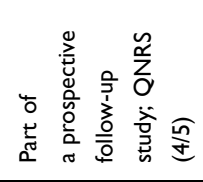 \\
\hline 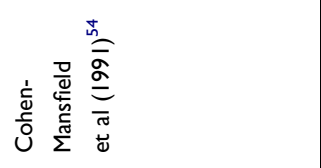 & 竞 & 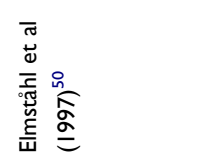 \\
\hline
\end{tabular}




\begin{tabular}{|c|c|c|}
\hline 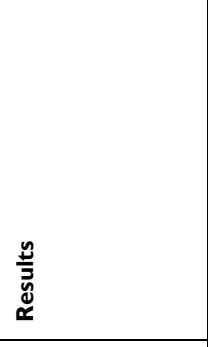 & 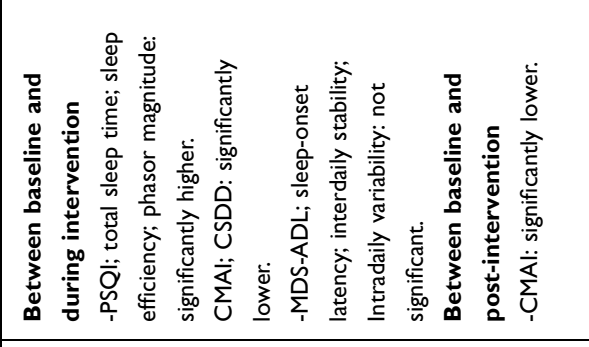 & 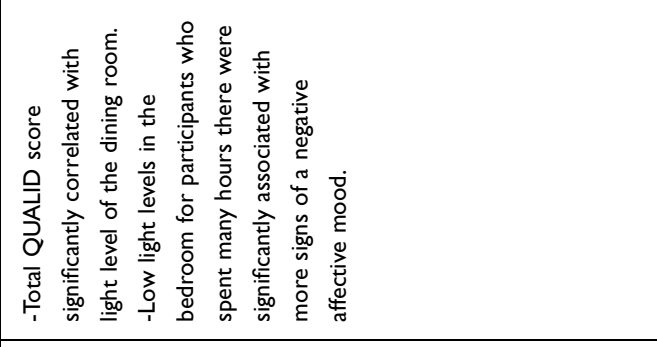 \\
\hline 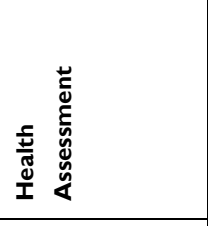 & 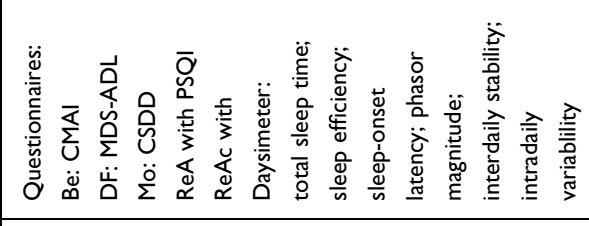 & 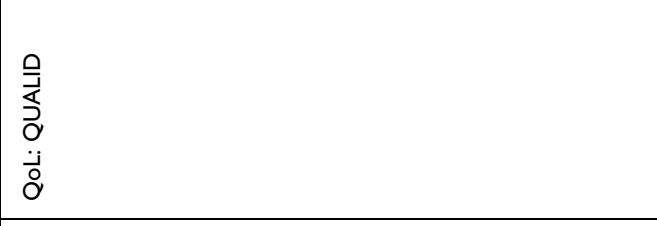 \\
\hline 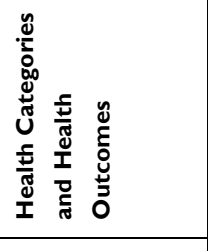 & 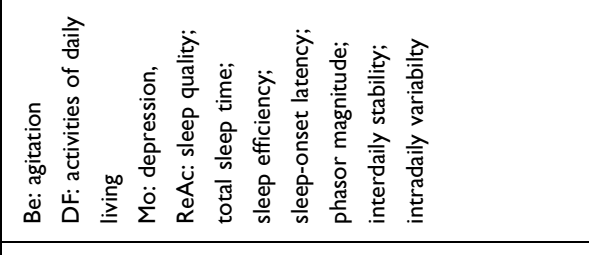 & 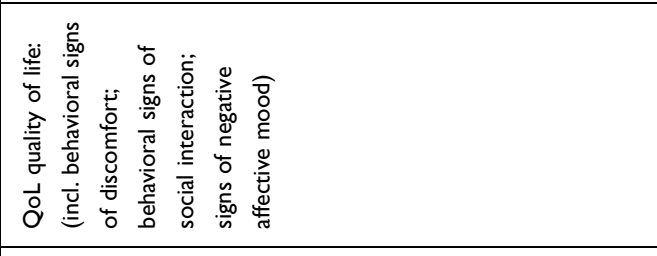 \\
\hline 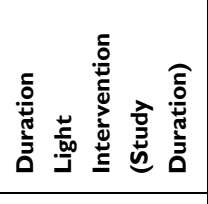 & 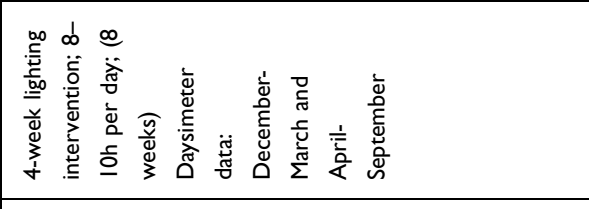 & 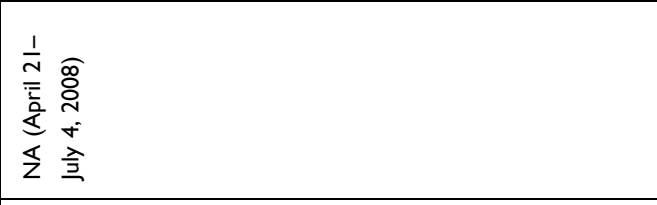 \\
\hline 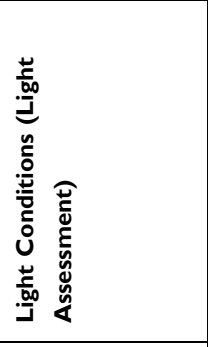 & 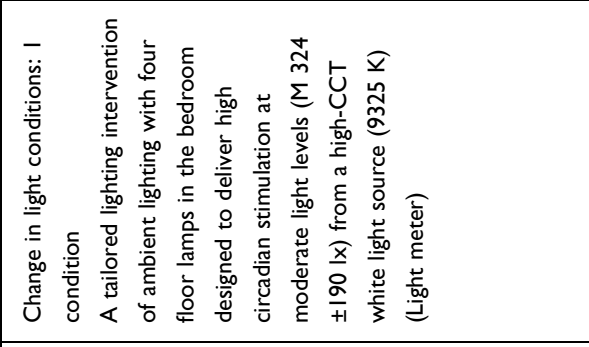 & 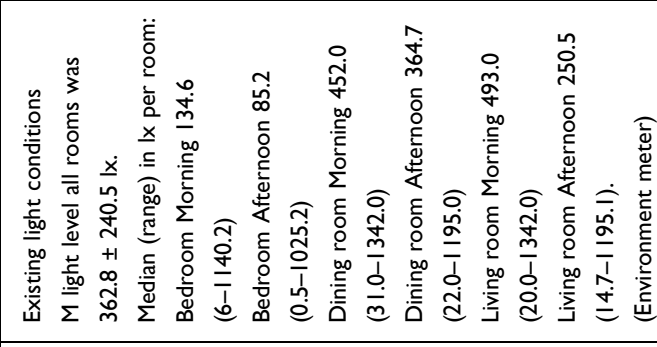 \\
\hline & 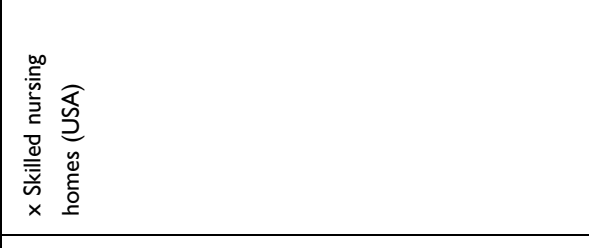 & 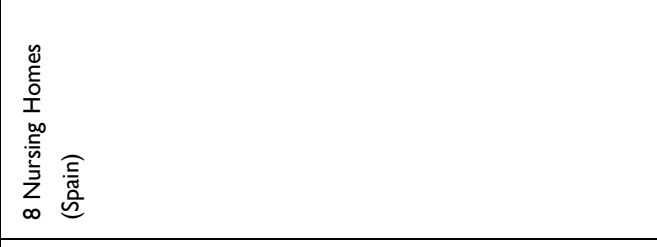 \\
\hline & 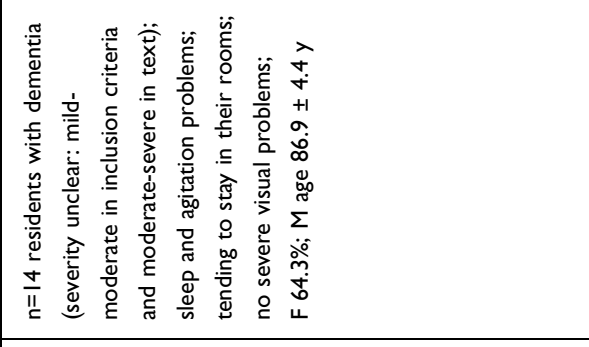 & 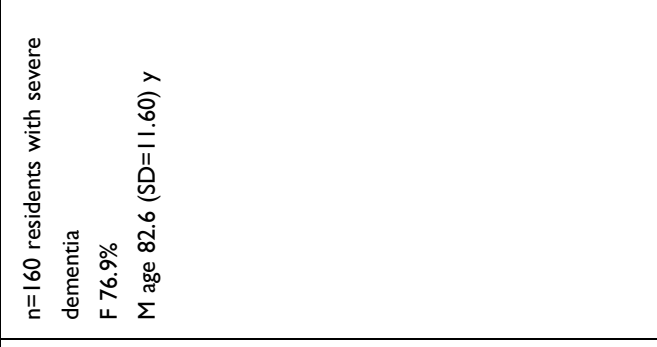 \\
\hline 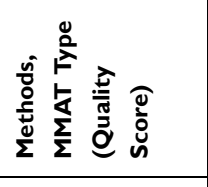 & 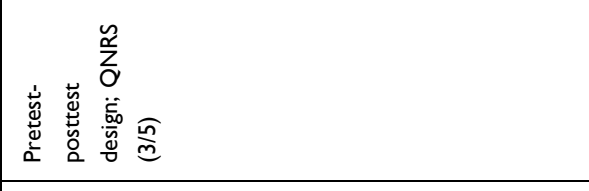 & 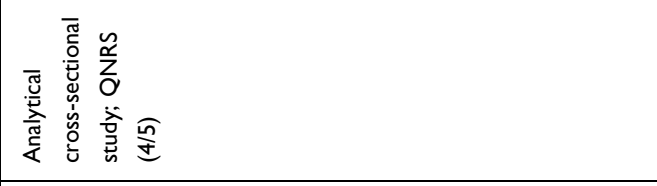 \\
\hline 辥 & 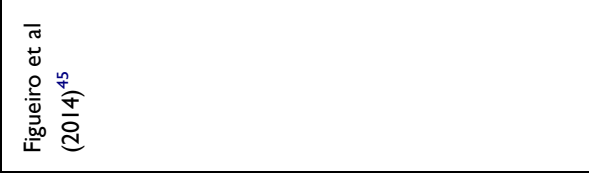 & 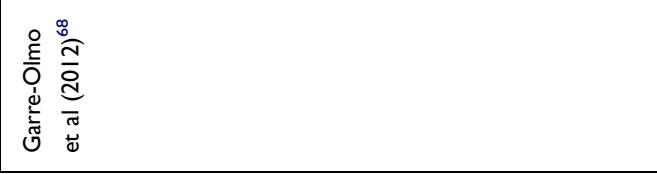 \\
\hline
\end{tabular}




\begin{tabular}{|c|c|c|}
\hline 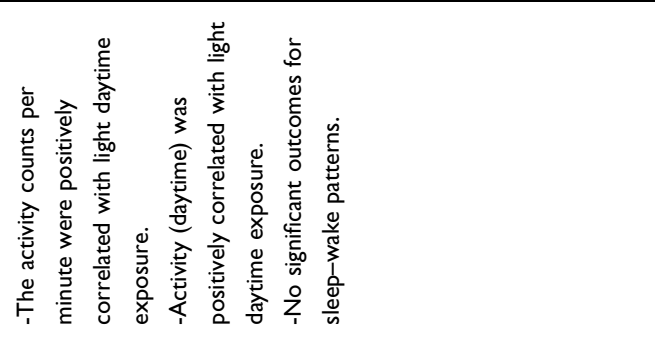 & 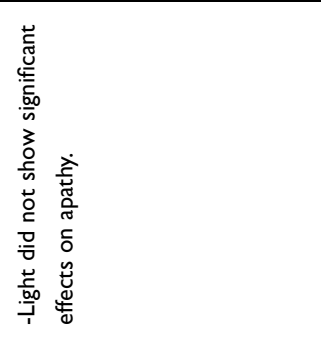 & 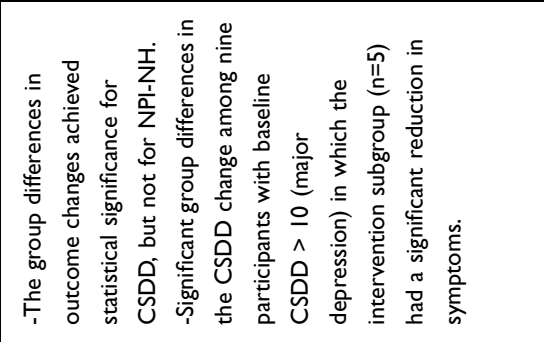 \\
\hline & 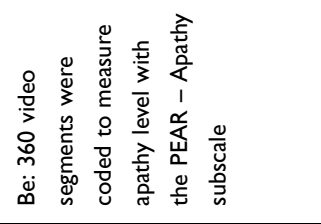 & 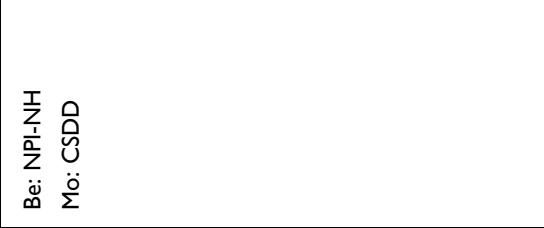 \\
\hline 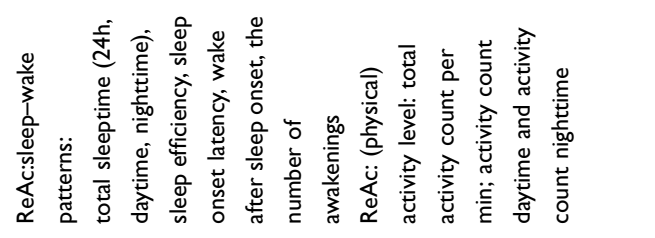 & 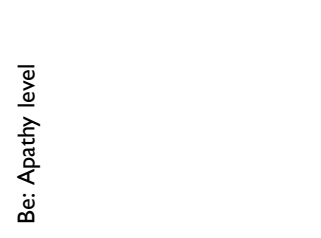 & 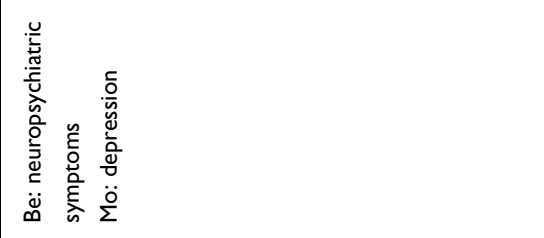 \\
\hline 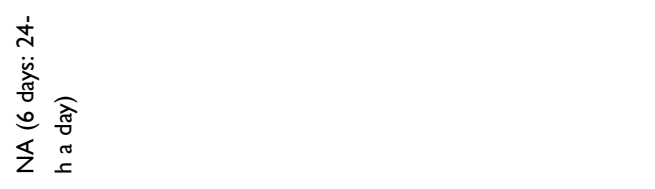 & 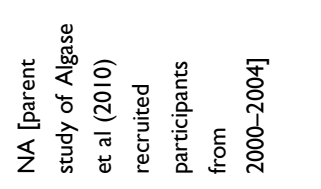 & 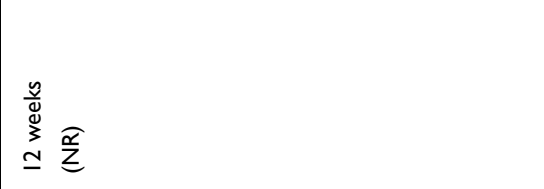 \\
\hline 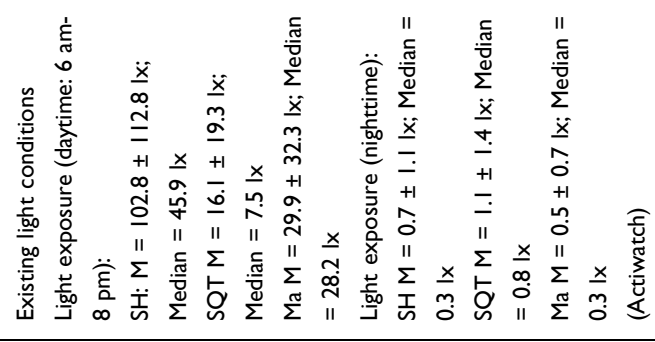 & 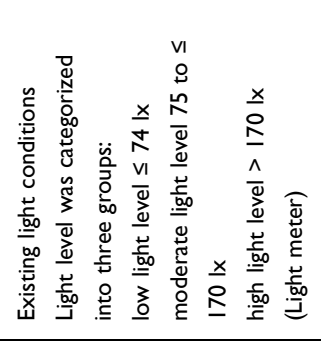 & 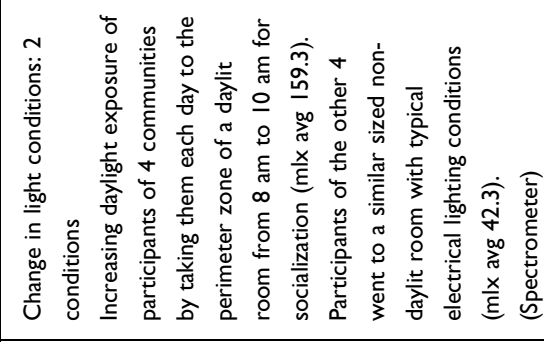 \\
\hline 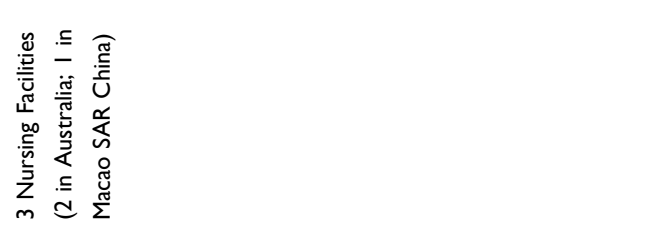 & 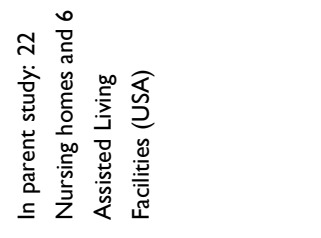 & 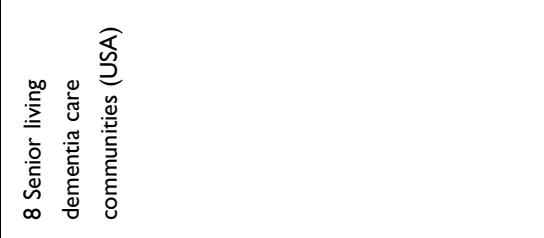 \\
\hline 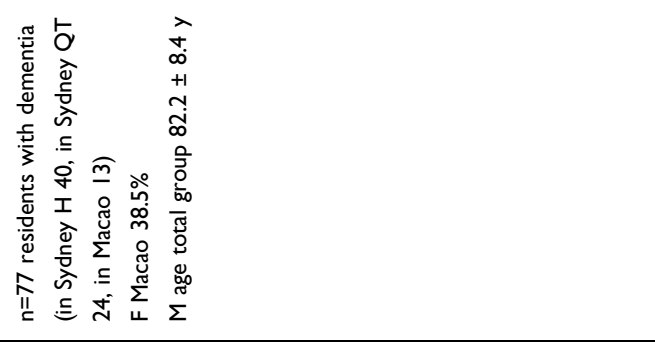 & 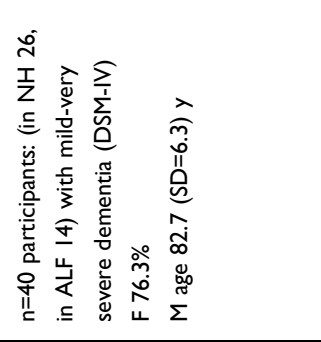 & 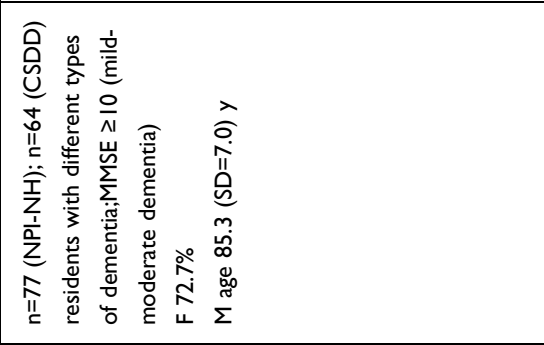 \\
\hline 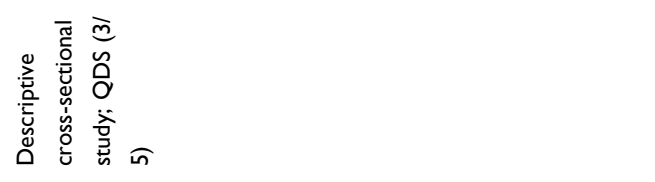 & 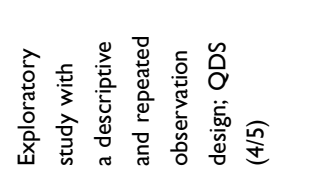 & 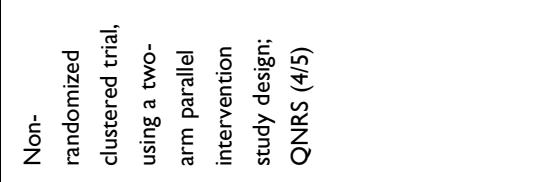 \\
\hline 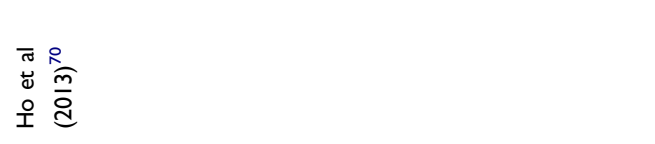 & 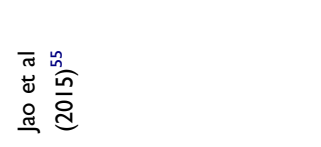 & 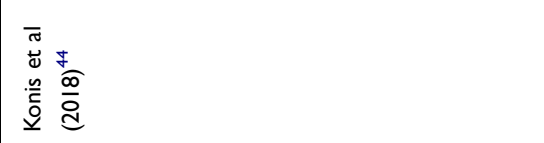 \\
\hline
\end{tabular}




\begin{tabular}{|c|c|c|}
\hline 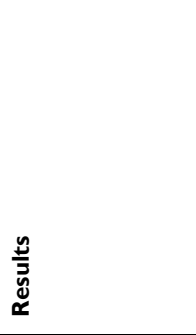 & 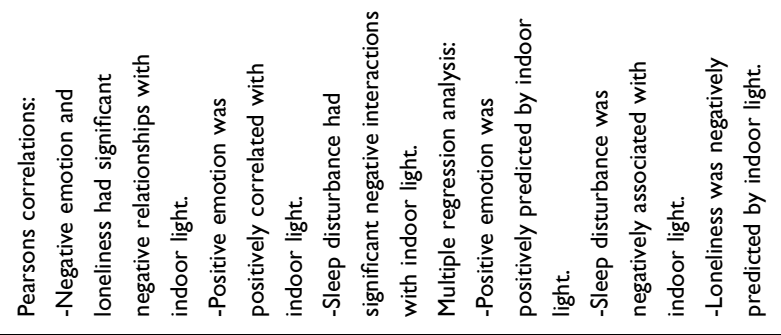 & 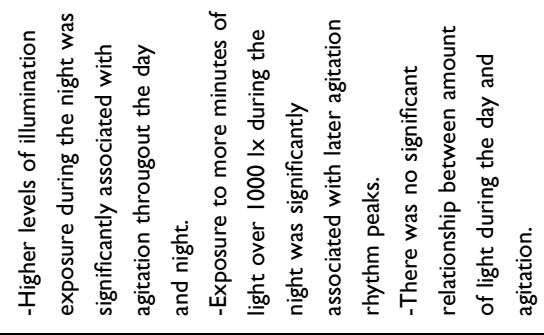 \\
\hline 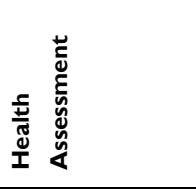 & 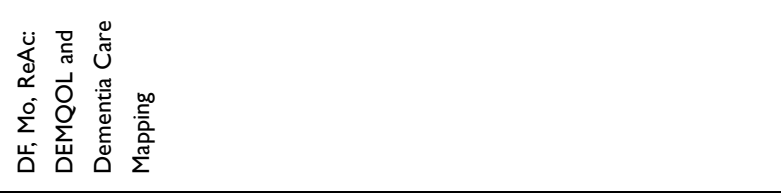 & 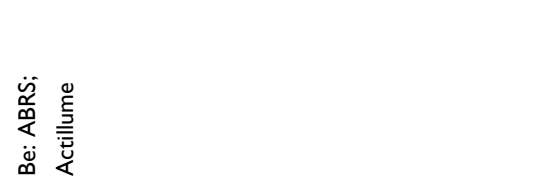 \\
\hline 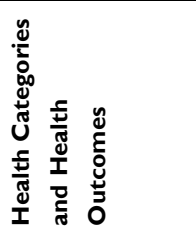 & 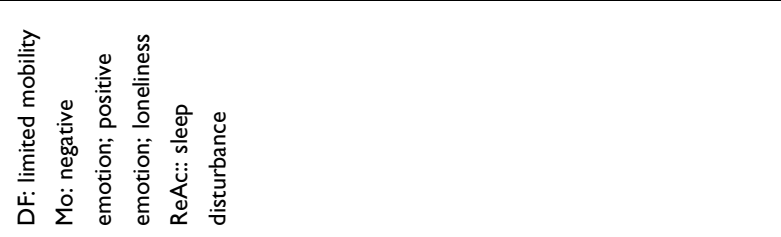 & 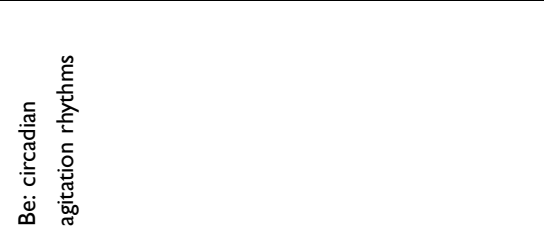 \\
\hline 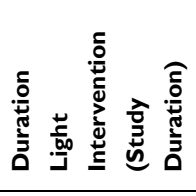 & 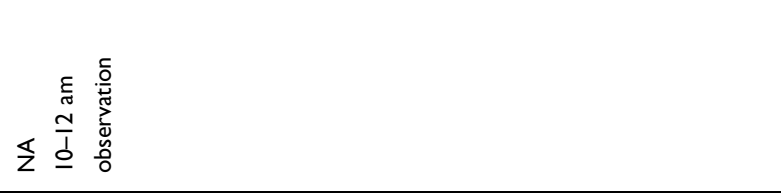 & 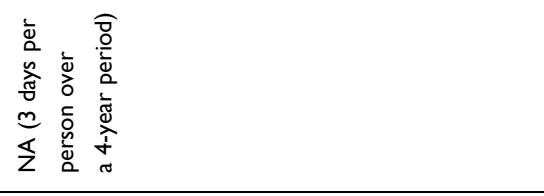 \\
\hline 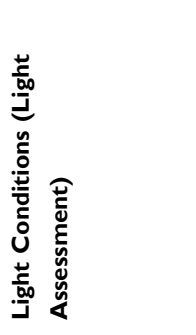 & 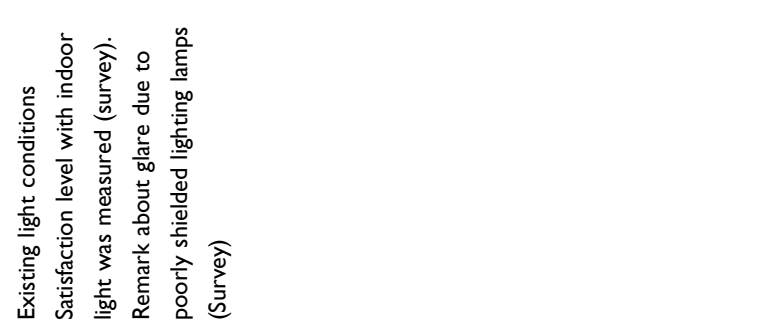 & 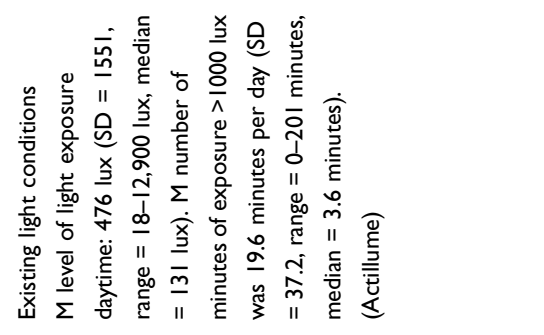 \\
\hline 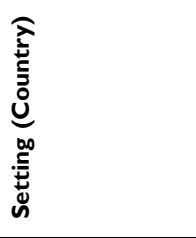 & 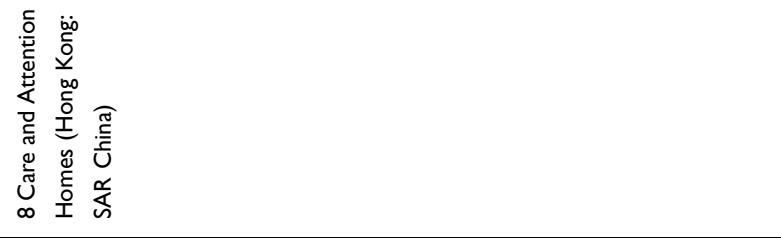 & 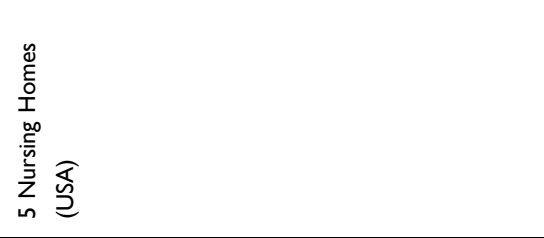 \\
\hline & 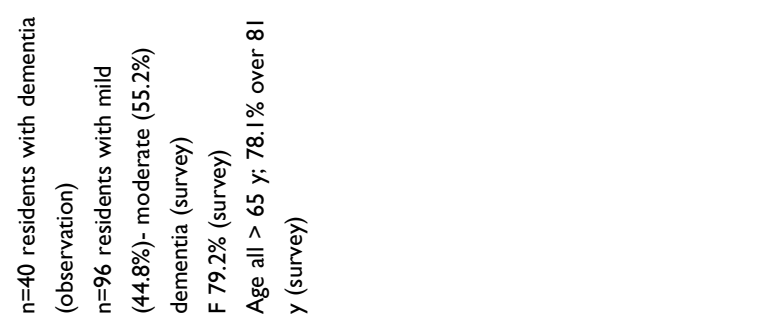 & 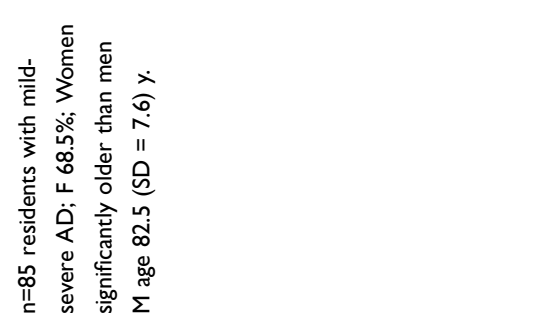 \\
\hline 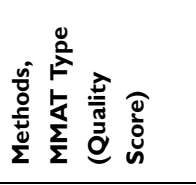 & 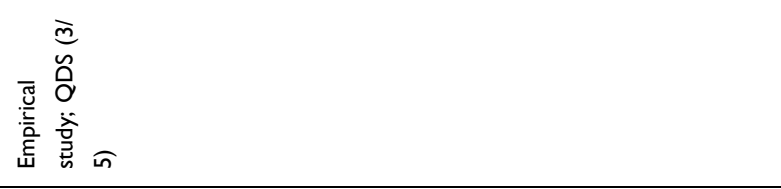 & 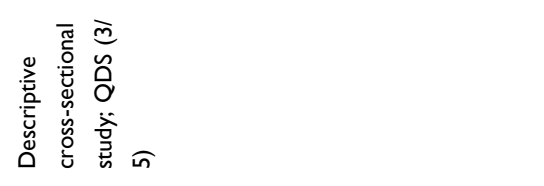 \\
\hline 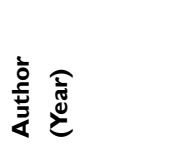 & 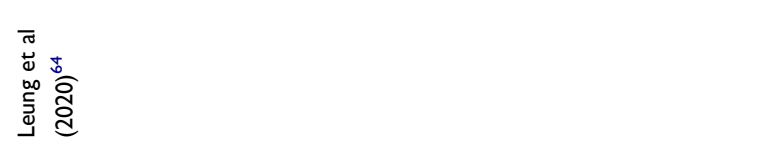 & 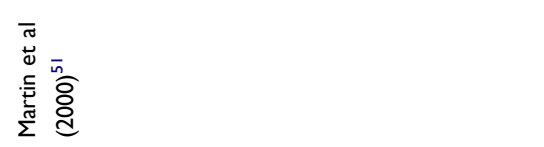 \\
\hline
\end{tabular}




\begin{tabular}{|c|c|}
\hline 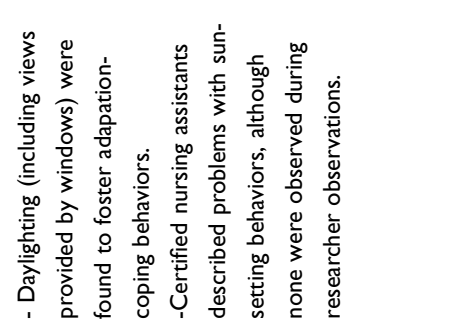 & 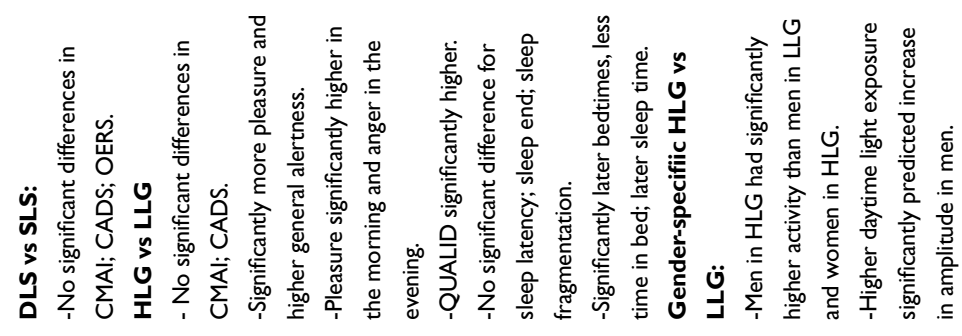 \\
\hline 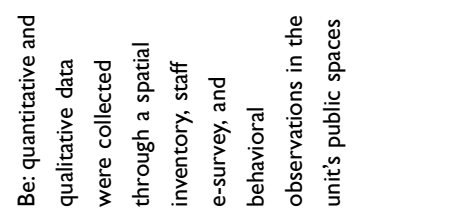 & 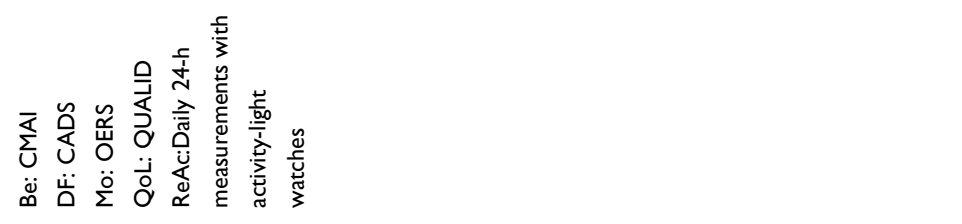 \\
\hline 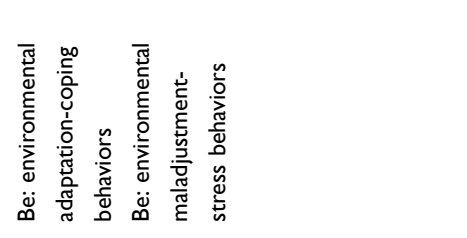 & 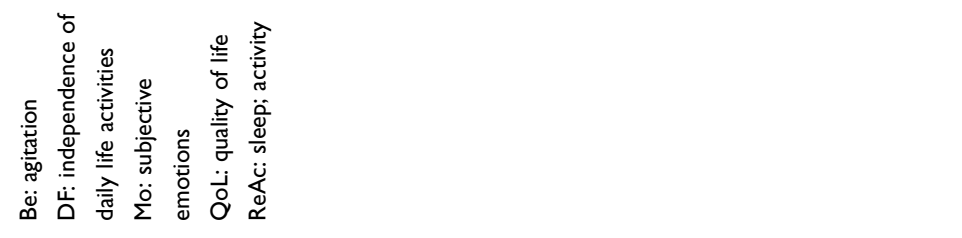 \\
\hline 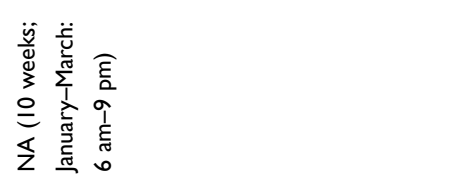 & 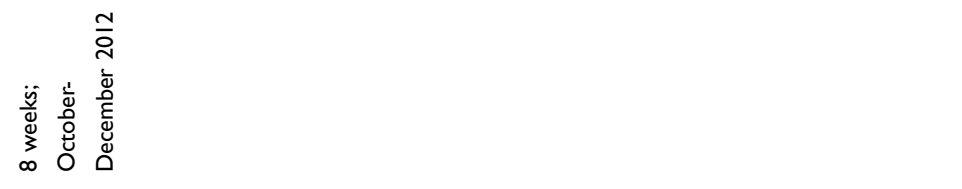 \\
\hline 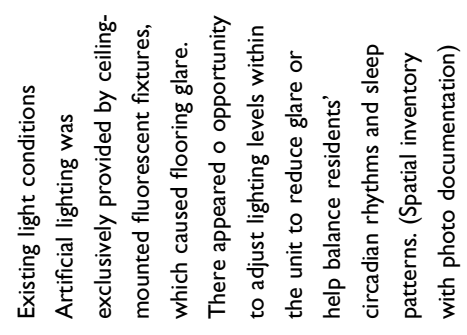 & 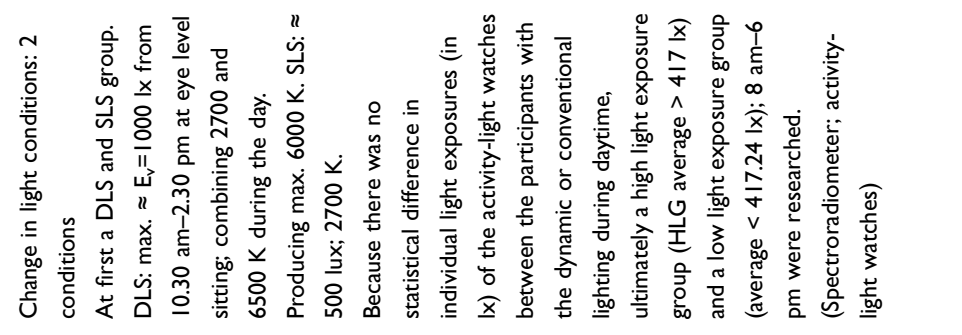 \\
\hline 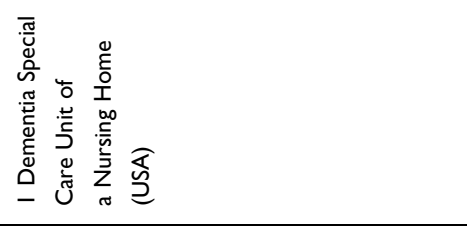 & 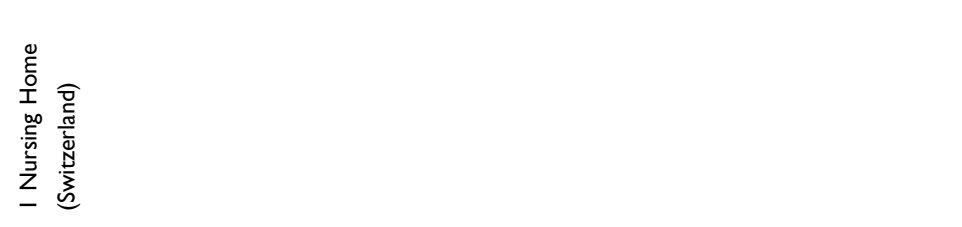 \\
\hline 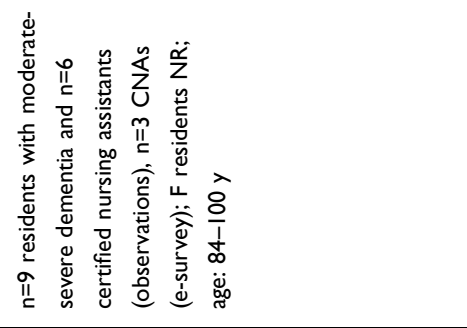 & 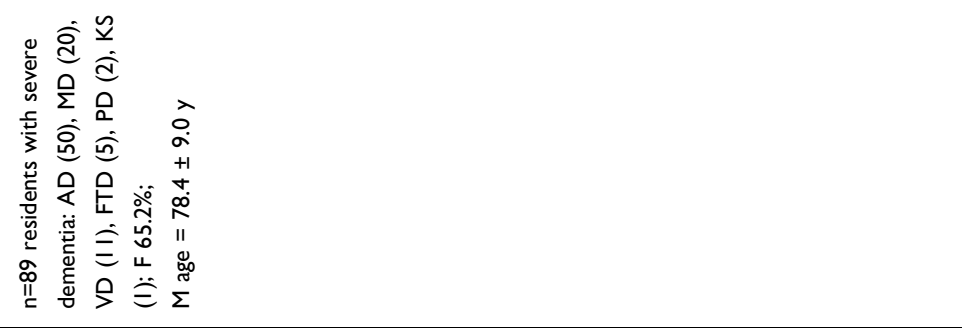 \\
\hline 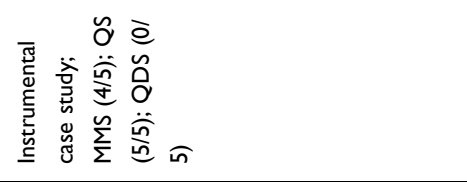 & 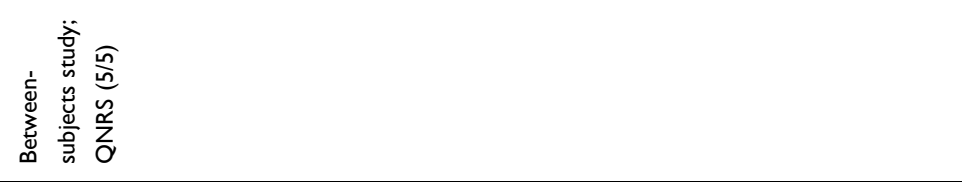 \\
\hline 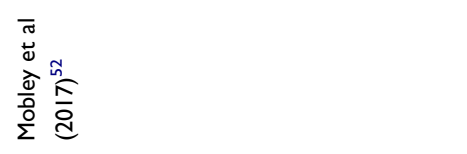 & 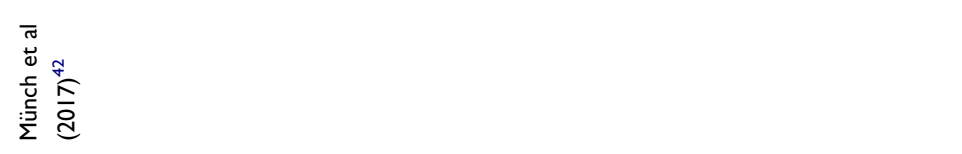 \\
\hline
\end{tabular}




\begin{tabular}{|c|c|c|}
\hline 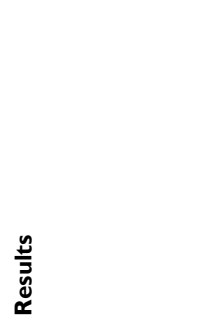 & 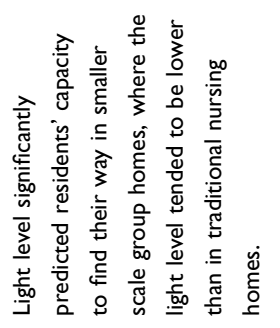 & 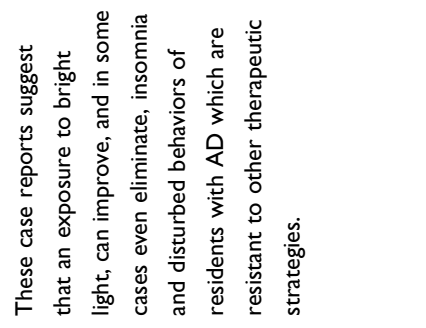 \\
\hline 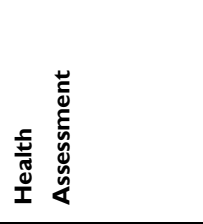 & $\begin{array}{l}\frac{Q}{Z} \\
\frac{1}{4} \\
\frac{\ddot{L}}{\Sigma} \\
\end{array}$ & 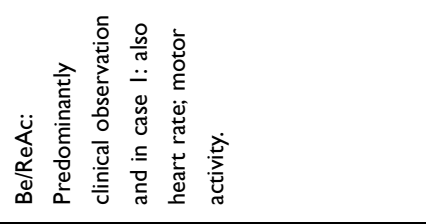 \\
\hline 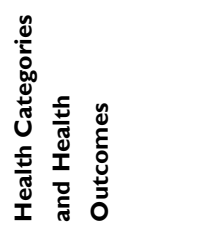 & 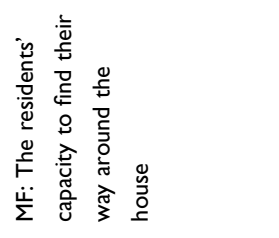 & 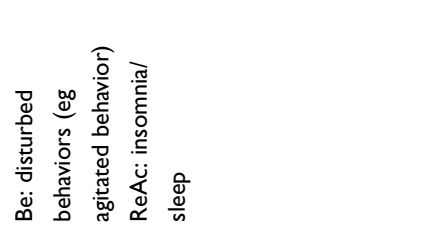 \\
\hline 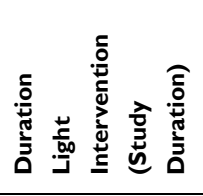 & 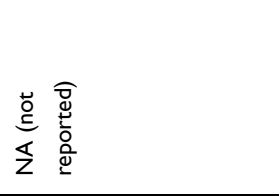 & 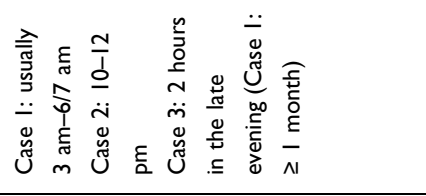 \\
\hline 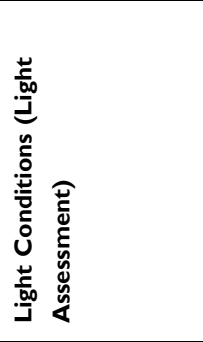 & 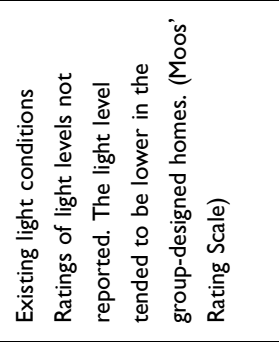 & 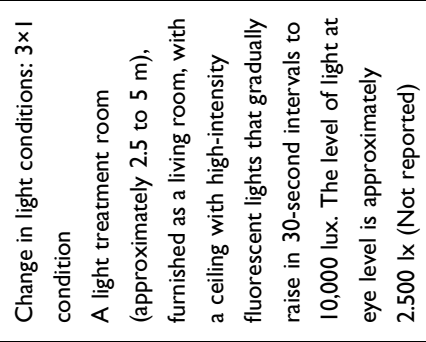 \\
\hline 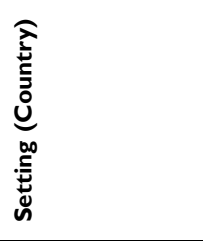 & 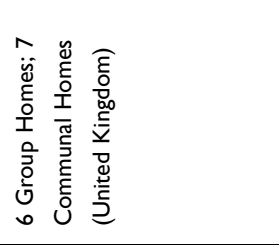 & 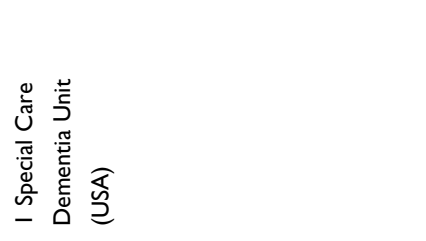 \\
\hline & 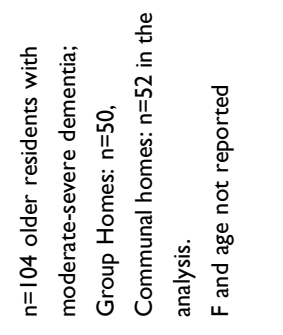 & 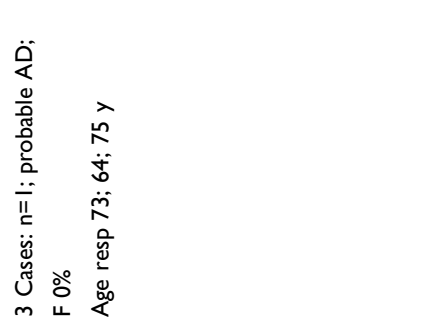 \\
\hline 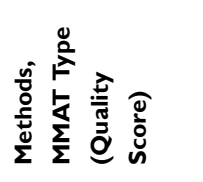 & 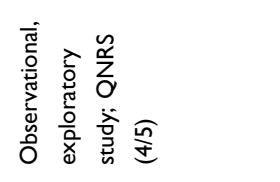 & 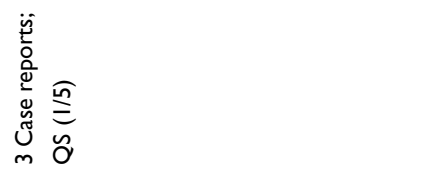 \\
\hline $\begin{array}{l}\text { 竞 } \\
\text { 妾 }\end{array}$ & 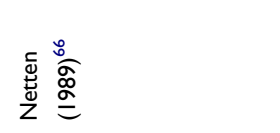 & 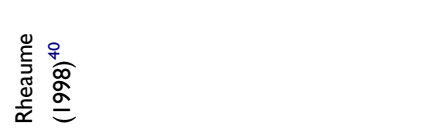 \\
\hline
\end{tabular}




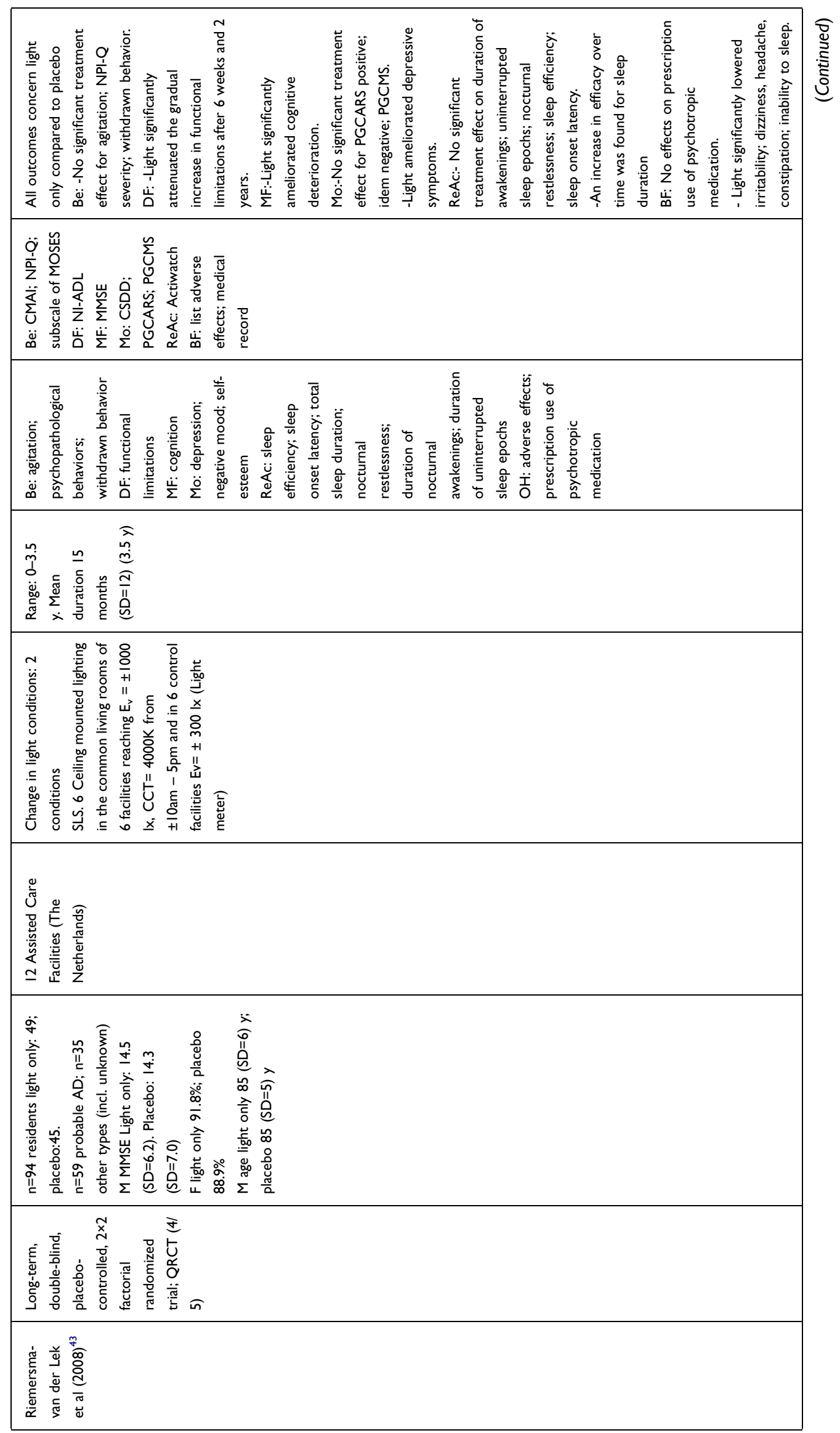




\begin{tabular}{|c|c|c|c|}
\hline $\begin{array}{l}\frac{n}{n} \\
\frac{\bar{z}}{x} \\
x\end{array}$ & 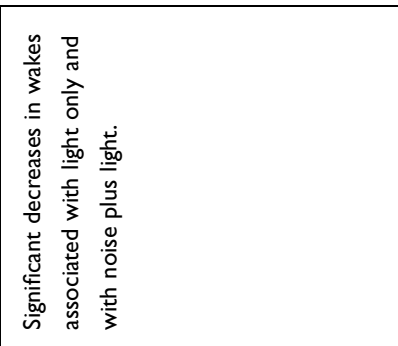 & 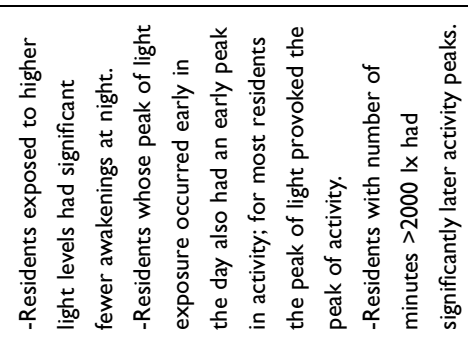 & 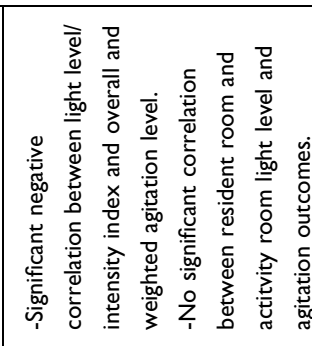 \\
\hline 吾 & 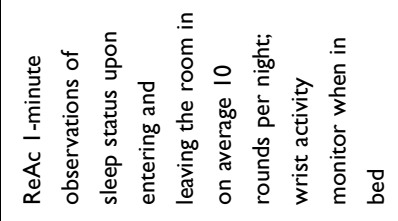 & 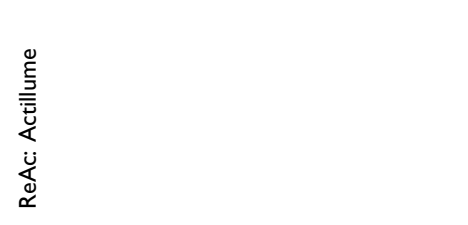 & 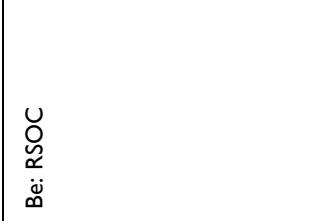 \\
\hline 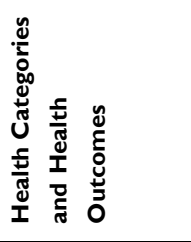 & 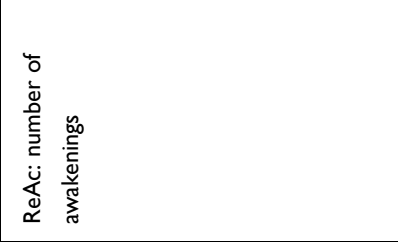 & 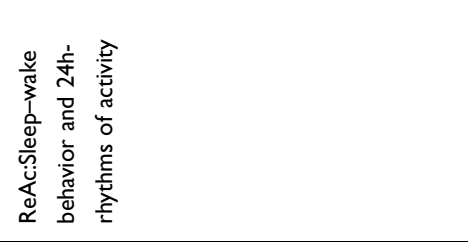 & 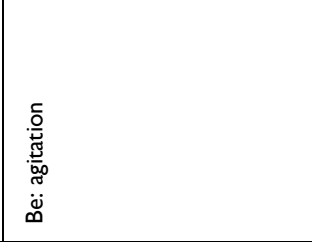 \\
\hline 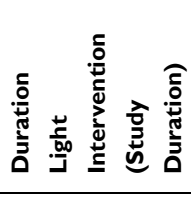 & 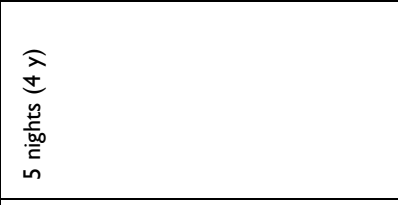 & 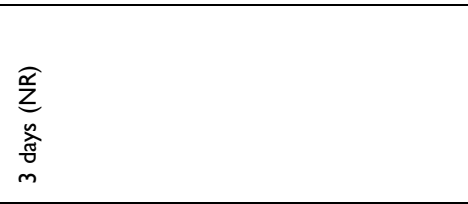 & 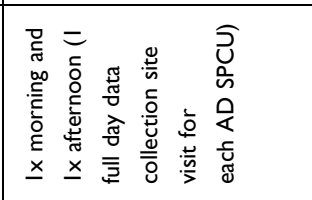 \\
\hline 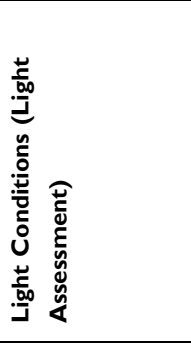 & 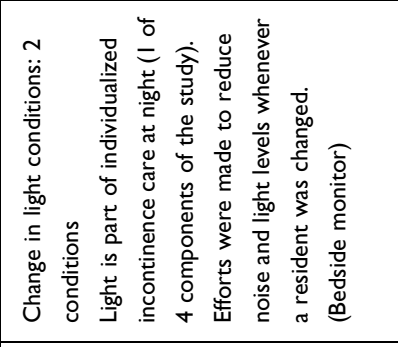 & 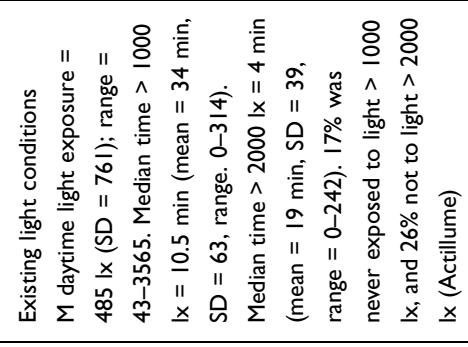 & 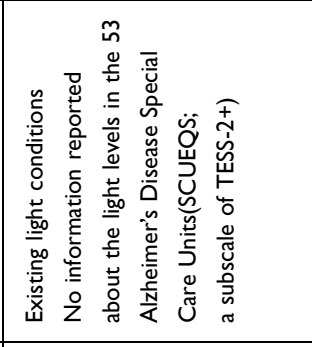 \\
\hline 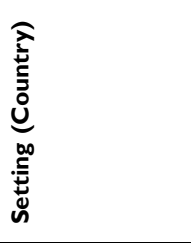 & 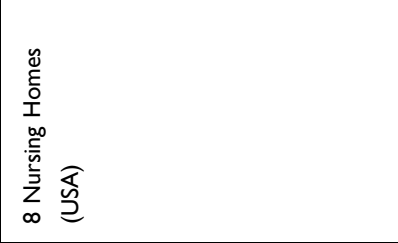 & 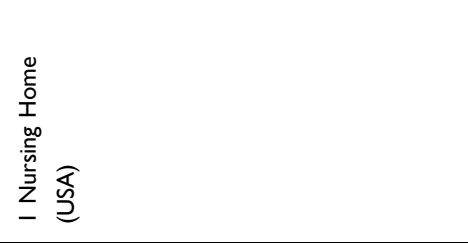 & 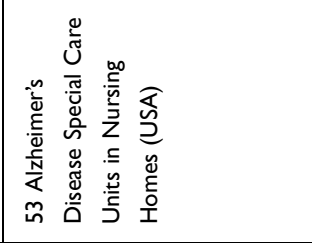 \\
\hline & 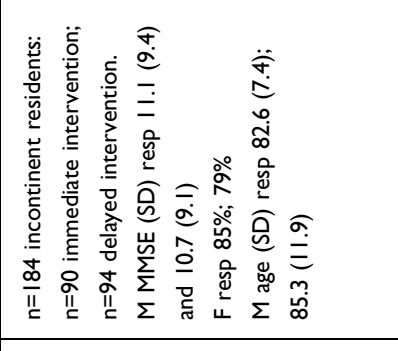 & 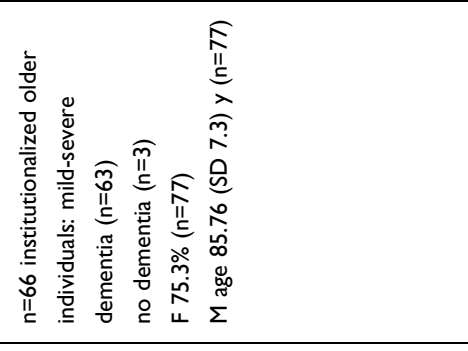 & 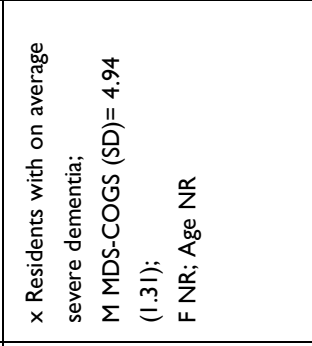 \\
\hline 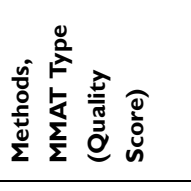 & 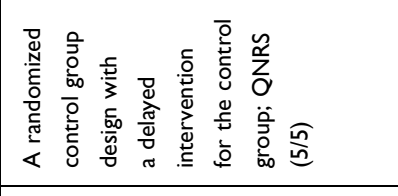 & 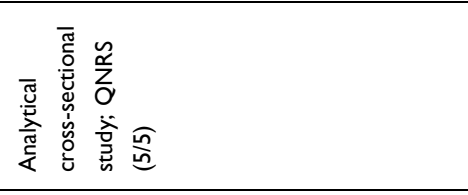 & 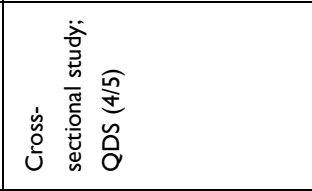 \\
\hline 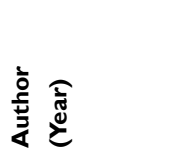 & 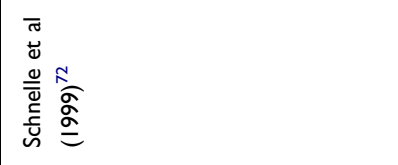 & 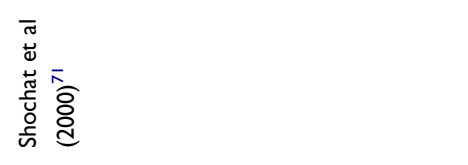 & 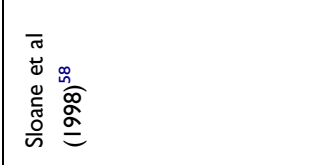 \\
\hline
\end{tabular}




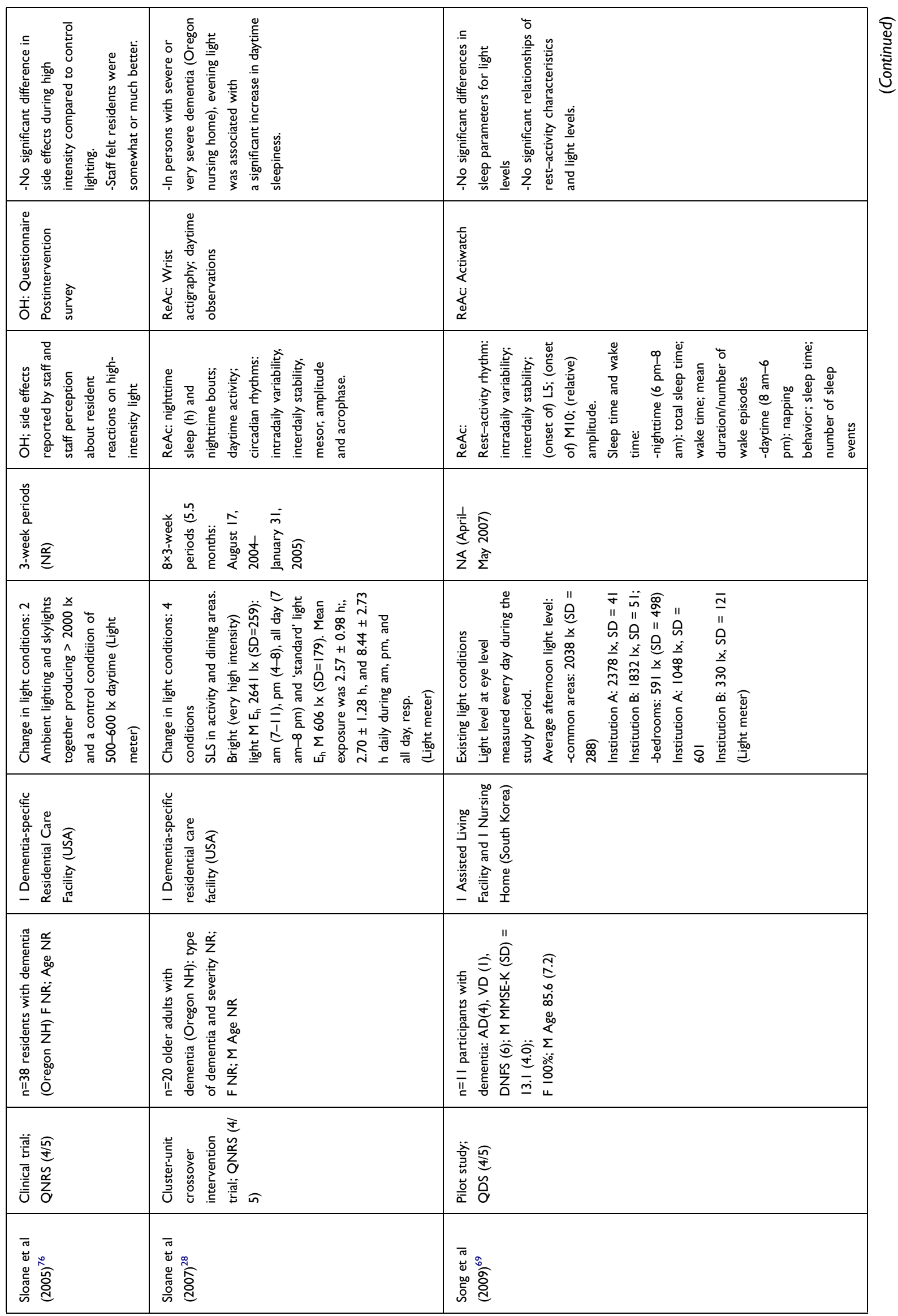




\begin{tabular}{|c|c|c|}
\hline $\begin{array}{l}\frac{\tilde{y}}{\vec{z}} \\
\stackrel{\bar{d}}{x}\end{array}$ & 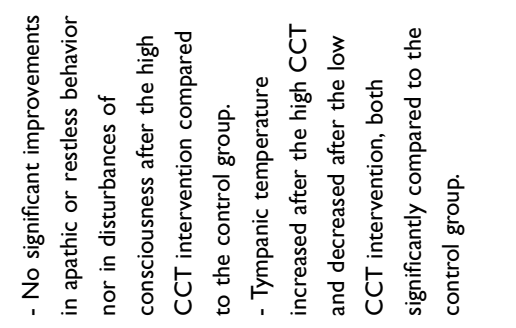 & 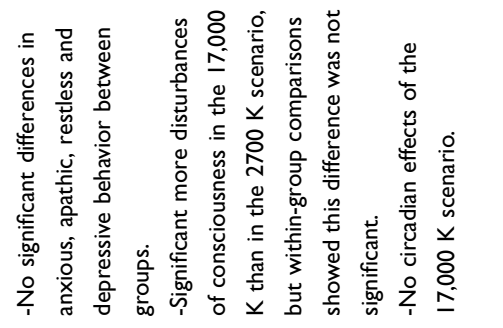 \\
\hline 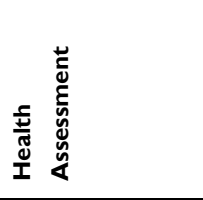 & 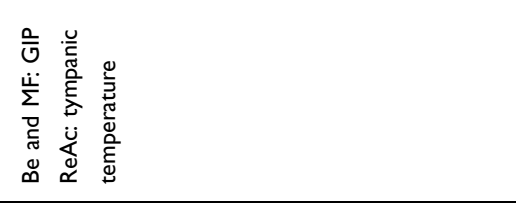 & 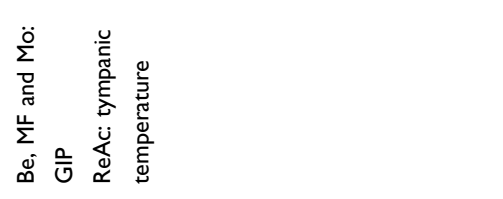 \\
\hline 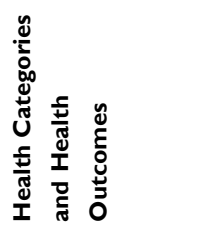 & 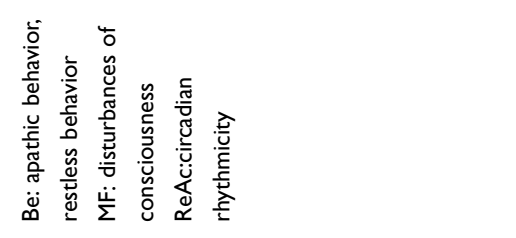 & 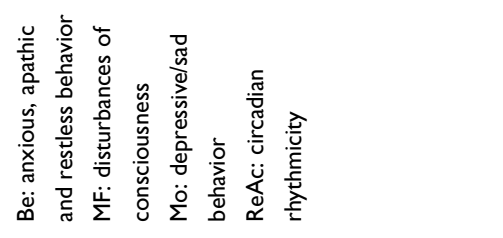 \\
\hline 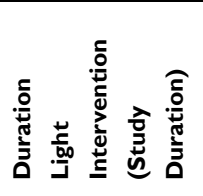 & 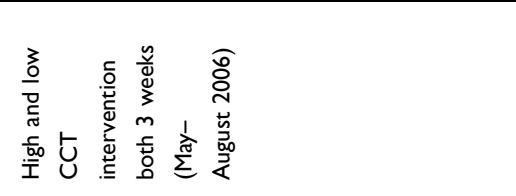 & 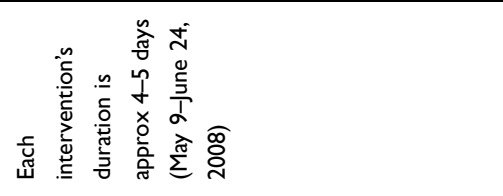 \\
\hline 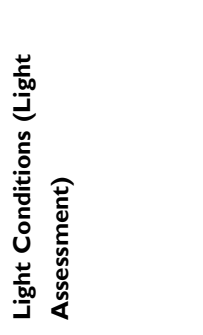 & 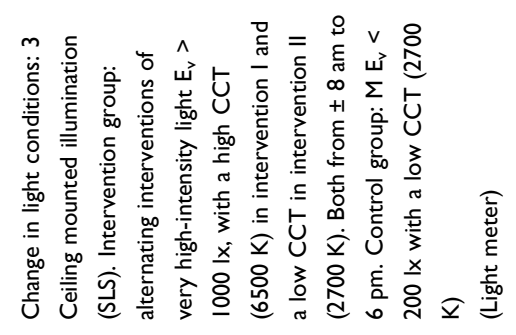 & 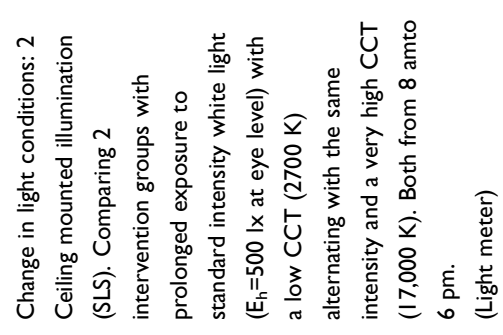 \\
\hline 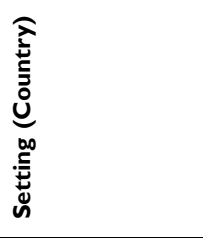 & 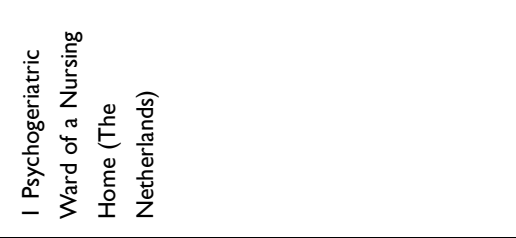 & 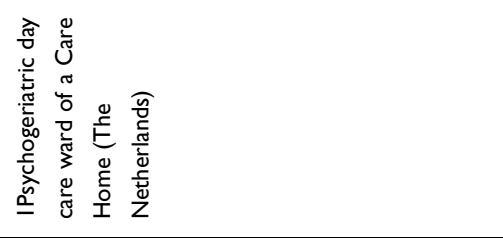 \\
\hline 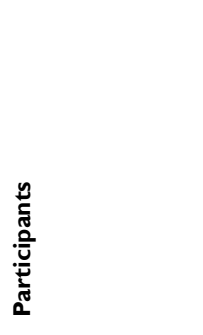 & 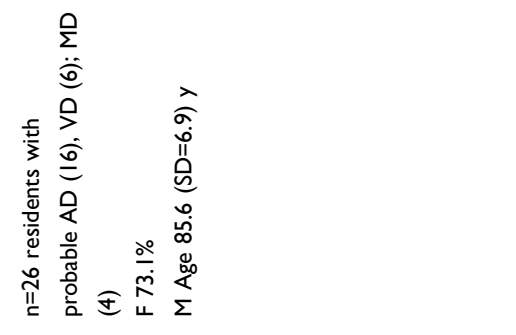 & 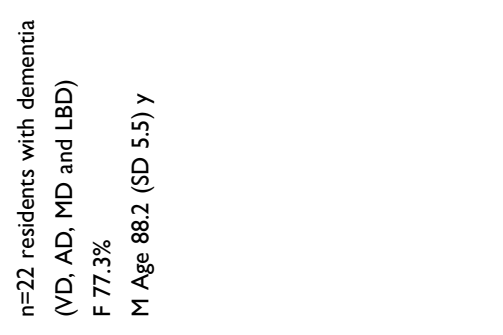 \\
\hline 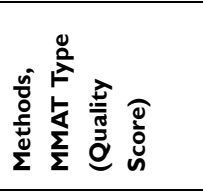 & 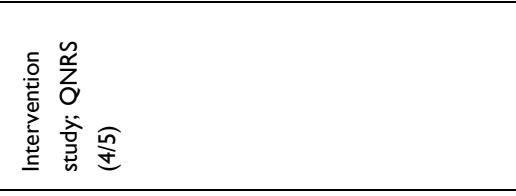 & 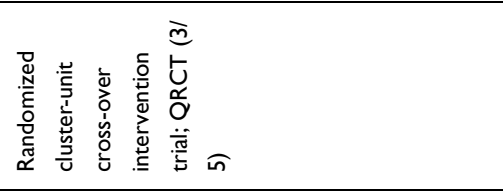 \\
\hline 旁㱏 & 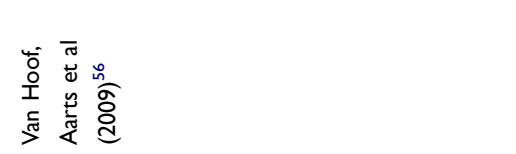 & 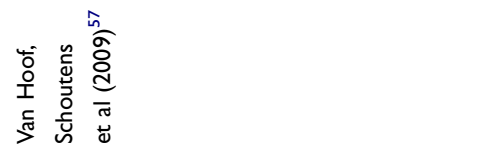 \\
\hline
\end{tabular}



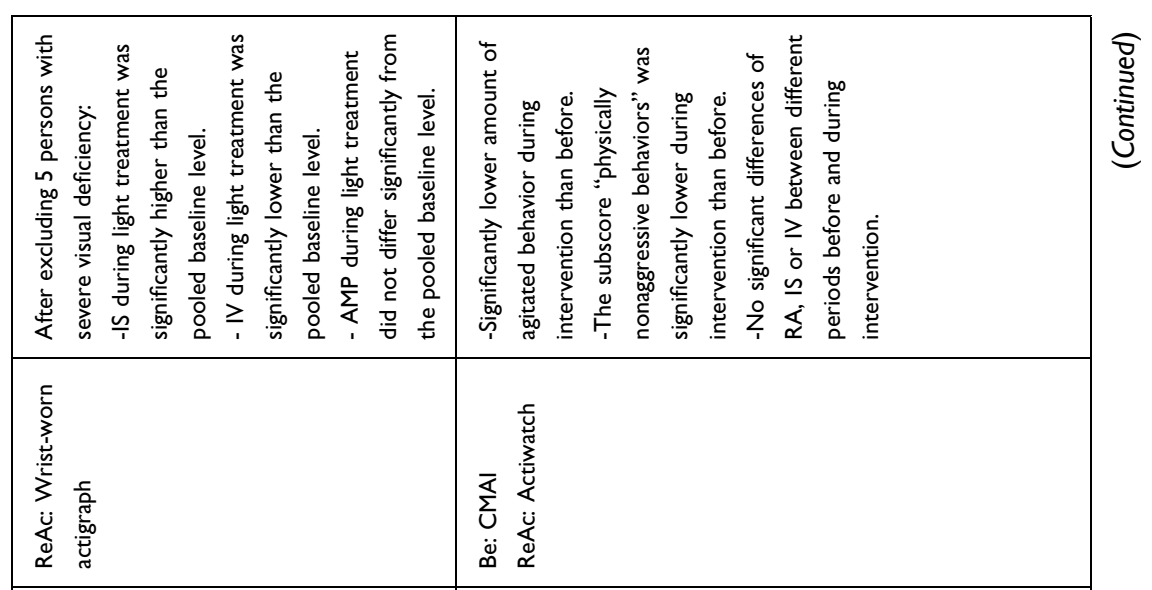

\begin{tabular}{|c|c|}
\hline 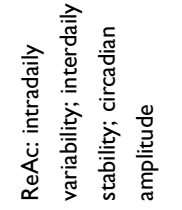 & 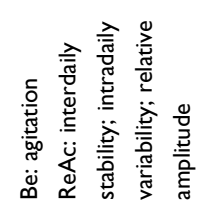 \\
\hline
\end{tabular}

\begin{tabular}{|c|c|}
\hline 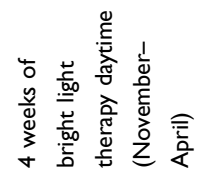 & 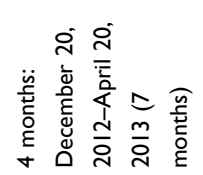 \\
\hline
\end{tabular}
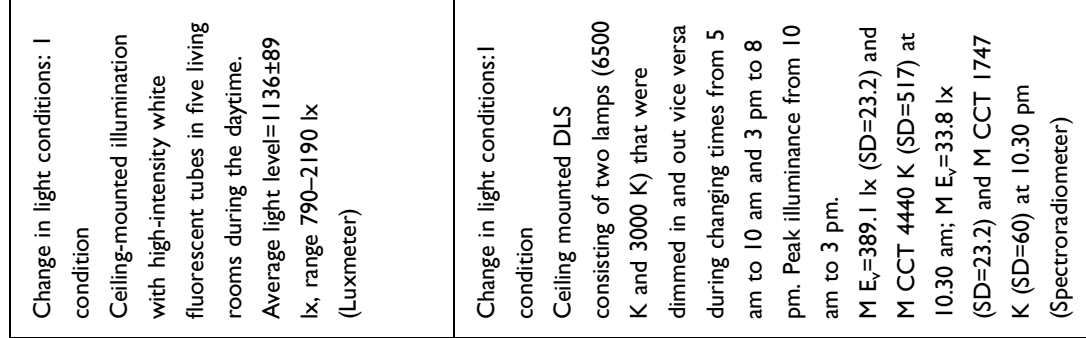

\begin{tabular}{|c|c|}
\hline 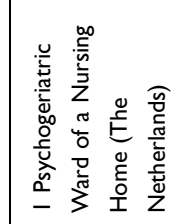 & 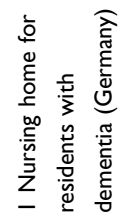 \\
\hline
\end{tabular}

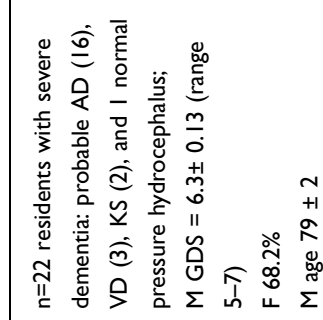

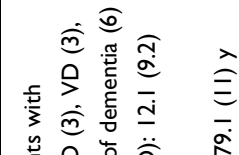

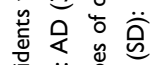

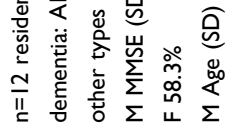

\begin{tabular}{|c|c|}
\hline 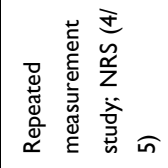 & 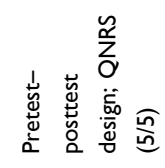 \\
\hline 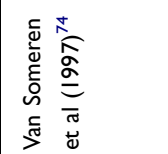 & 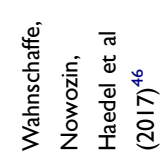 \\
\hline
\end{tabular}




\begin{tabular}{|c|c|c|c|}
\hline 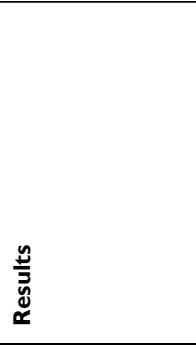 & 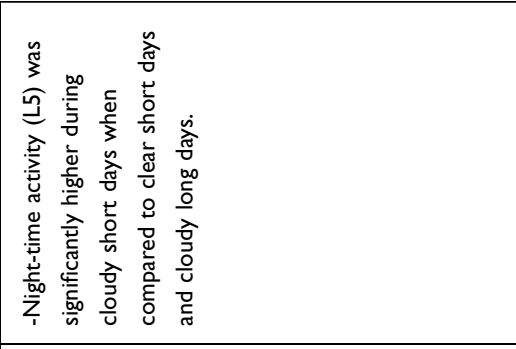 & 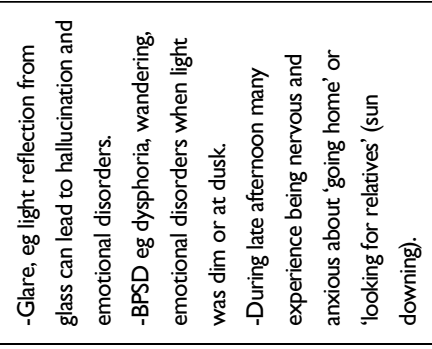 & 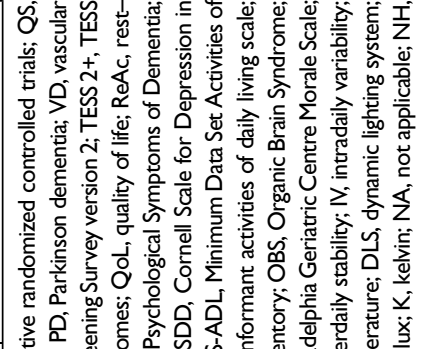 \\
\hline 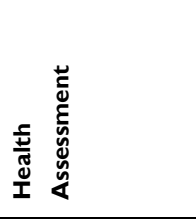 & & 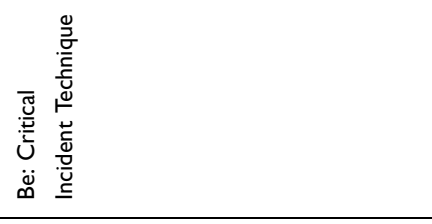 & 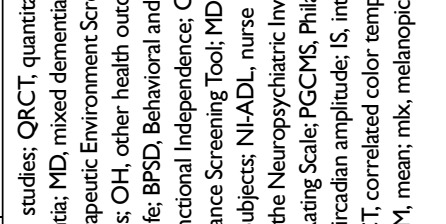 \\
\hline 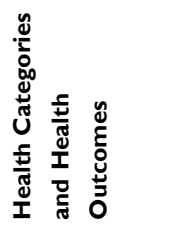 & 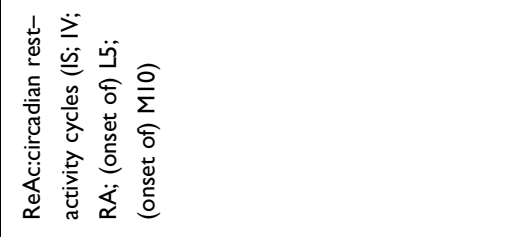 & 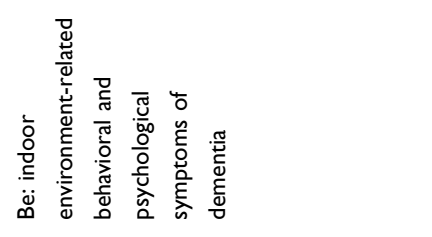 & 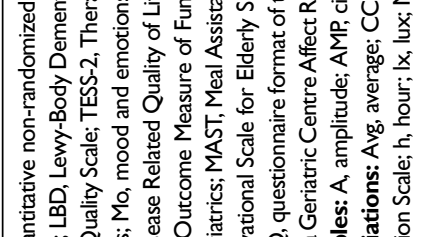 \\
\hline 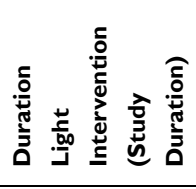 & 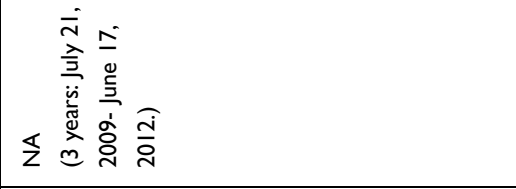 & 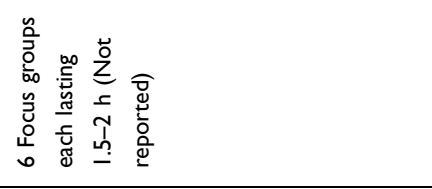 & 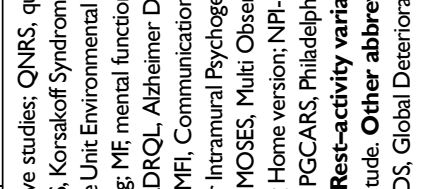 \\
\hline 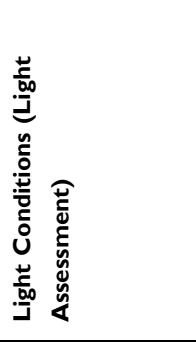 & 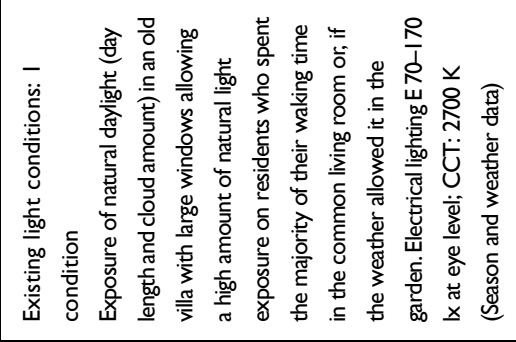 & 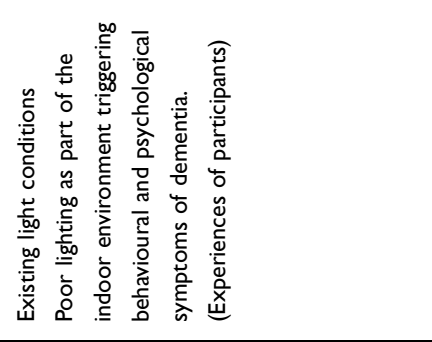 & 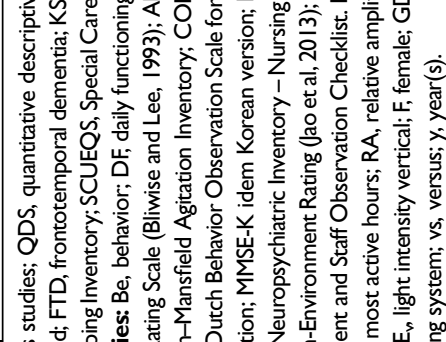 \\
\hline 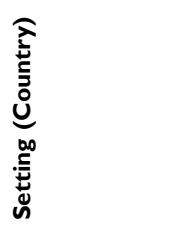 & 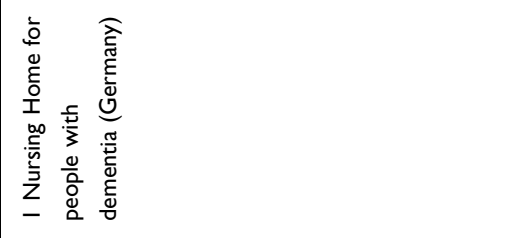 & 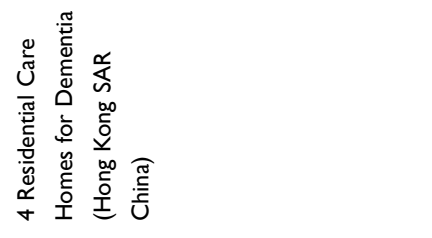 & 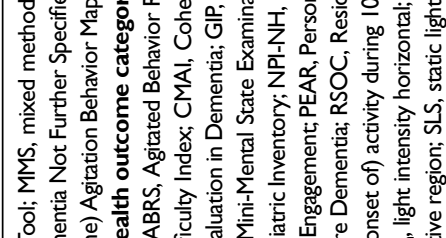 \\
\hline & 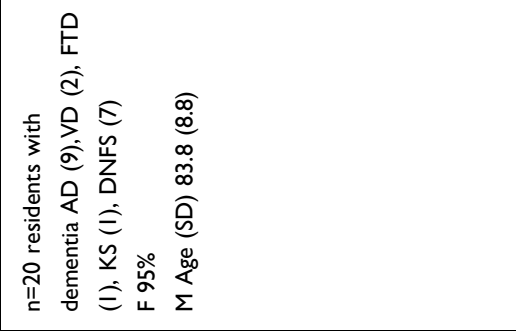 & 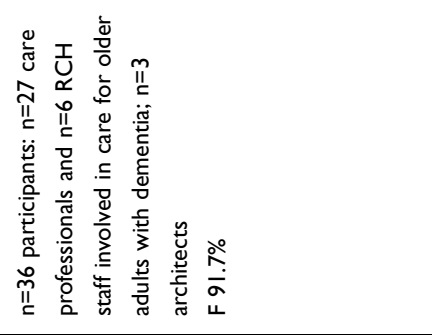 & 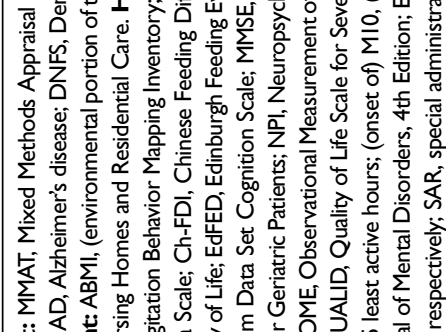 \\
\hline 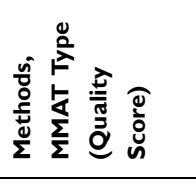 & 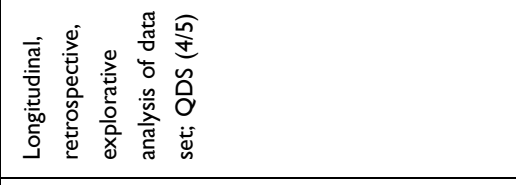 & 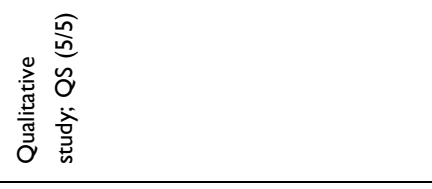 & 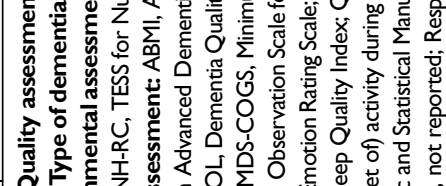 \\
\hline 童害 & 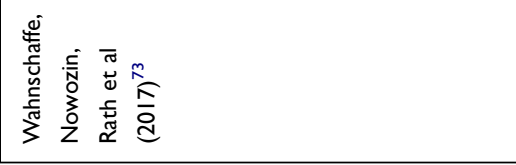 & 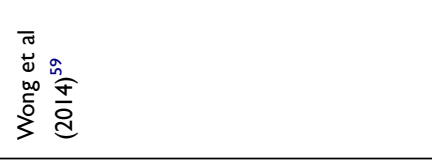 & 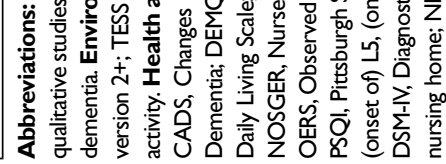 \\
\hline
\end{tabular}


Table 3 Characteristics of Light

\begin{tabular}{|l|l|}
\hline Light Level (Illuminance) in lux (Ix) & CCT in kelvin (K) \\
\hline Very low intensity $<50$ & Warm (white) light 2700-3200 \\
Low intensity $50-200$ & Neutral (white) light 3200-4200 \\
Moderate intensity 200-400 & Cool (white) light 4200-6200 \\
Standard intensity 400-600 & Very cool (blue) light 6200-9500 \\
High intensity 600-1000 & Extremely cool (blue) light $>9500$ \\
Very high intensity $>1000$ & \\
\hline
\end{tabular}

Notes: These light levels can be measured horizontally or vertically. Abbreviation: CCT, correlated color temperature.

physically nonaggressive behaviors. ${ }^{46}$ In addition to agitation assessed by staff caregivers with the CMAI, the study of Barrick et $\mathrm{al}^{27}$ also used direct observations of research personnel to evaluate agitation. Analyzing these observations, nursing home participants with moderate dementia were significantly more agitated under all three bright light conditions (7-11 am, 4-8 pm and all day $7 \mathrm{am}-8 \mathrm{pm}$ ) than in standard light conditions. Therefore, Barrick et $\mathrm{al}^{27}$ noted that very high-intensity cool light may even exacerbate agitation. An individually timed light intervention simulating a natural pattern of dusk and dawn did not have an effect on agitation. ${ }^{47}$

Most studies regarding existing light conditions $(n=5$; $71.4 \%$ ) did not find a relationship between light during the day and sets of challenging behaviors. ${ }^{48-52}$ However, one of these studies investigating agitation rhythms found a relationship of agitation and very high-intensity light during the night. ${ }^{51}$ Participants with more minutes of exposure to very high-intensity light during the night displayed their agitation later in the day. They also found that exposure to higher light levels during the night resulted in experiencing some agitation throughout the day and night, with no consistent periods without agitation. Possible sources of the nocturnal light exposure were lights being left on in the bedrooms or participants wandering over to a brightly lit nurses' station. ${ }^{51}$

Six articles reported on one or more distinct challenging behaviors, namely anxious behaviors, apathy, pacing, restless behavior, wandering behavior and withdrawn behavior. $^{43,53-57}$ Only two of these studies (33.3\%) found the behaviors to have a significant relationship with indoor light. These quantitative descriptive studies conducted in existing light conditions investigated physically active challenging behaviors. The concerned studies reported significantly less wandering ${ }^{53}$ or pacing, ${ }^{54}$ when light levels were low, which is contrary to findings in similar light conditions on sets of challenging behaviors. ${ }^{58,59}$
Two studies investigated a direct influence of light on positive behaviors, namely adaptation-coping and social behavior. A mixed methods study found that indoor daylight improved adaptation-coping behaviors. Residents seemed to orient strolling behaviors toward areas with increased daylight and frequently paused to look through the glass in two exit doors. ${ }^{52}$ Using a crossover design, a dawn-dusk simulation did not produce significant effects on social behavior. ${ }^{47}$

\section{Indirect Influence of Light on Behavior}

Six studies reported on the relationship of indirect aspects of light exposure and sets of challenging behaviors. Most studies $(n=4 ; 66.7 \%)$ found no significant temporal aspects regarding sets of challenging behaviors. They did not find any significant effects in these behaviors before, during or after sunset, nor for any parts of the day, different days of the week, daylengths, months or seasons. ${ }^{27,51,52,60}$

With regard to sundowning behavior, the results depended on the perspective the observations were made from. Whereas care professionals mentioned that persons with dementia showed (more) challenging behaviors around sunset or in the evening, ${ }^{52,59,61}$ none of these behaviors were observed during researchers'

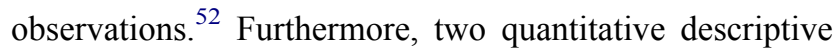
studies that specifically focused on the timing of these challenging behaviors and used researcher assessments, found no significant proof of these behaviors during or after sunset either. ${ }^{51,60}$ Bliwise et al ${ }^{60}$ stated, therefore, that these disruptive behaviors might occur with identical frequency throughout the day, but might have more impact on the nursing staff later in the day. The study focusing on agitation rhythms showed a substantial amount of variation in these rhythms. ${ }^{51}$ This study identified only two persons with dementia $(2.4 \%)$ having an agitation peak at sundown.

\section{Daily Functioning}

Studies investigating the impact of indoor light on daily functioning of persons with dementia focused on independence in activities of daily living (ADL), aspects of food intake and, mobility or falls. A randomized controlled trial (RCT) examining the effectiveness of a long-term intervention (up to 3.5 years) of high-intensity neutral light showed that the ADL in the intervention group decreased significantly less compared to the control group. ${ }^{43}$ Two studies using different, but short-term (4 and 8 weeks) light interventions during the day did not produce 
significant effects on ADL compared to baseline ${ }^{45}$ or the control group. ${ }^{42}$ In the study of Münch et al, ${ }^{42}$ the actual 24-h light exposure of persons with dementia was measured using activity watches with a light sensor. This study, comparing the influence of a dynamic lighting system to standard lighting, did not find any significant differences in the average daily light exposure between the groups. After dividing the sample into a high light and a low light group, based on their actual light exposure, the researchers did not find any significant differences in ADL either. A short-term dawn-dusk light intervention (8 weeks) also did not have a significant impact on ADL. ${ }^{47}$

Regarding food intake, beneficial effects of light have been found. A cross-sectional study, examining the influence of existing light conditions, showed a significant negative association of indoor light with food intake difficulties during dinnertime, but not during lunchtime. ${ }^{62} \mathrm{An}$ intervention with improved lighting and table setting contrast during breakfast, lunch and dinner increased 3-day caloric intake of almost all participants. ${ }^{63}$ This beforeafter study also showed higher functioning during meals.

Investigating the relationship of light and falls, a crosssectional study using an environmental assessment tool (Table 2) found that light glare was significantly correlated with a higher risk of falls, while light intensity and light evenness were not. ${ }^{48}$ A quantitative descriptive study found that satisfaction with indoor light had no significant correlation with limited mobility. ${ }^{64}$

\section{Mental Functions}

Seven articles reported on different kinds of mental functions, including disturbances of consciousness, spatial orientation, cognition, memory and engagement. No significant effects were found of the two very different light interventions on disturbances of consciousness compared to the control condition. ${ }^{56,57}$ Studies in existing light conditions demonstrated indoor light being an important aid for spatial orientation of persons with dementia. ${ }^{52,61,65,66}$ Observations showed that poorly lighted areas were avoided by people with dementia, thus creating overcrowding in other areas. ${ }^{61,65}$ In a qualitative study, men were observed to urinate in the "slop hopper" because, unlike the toilet areas, this area was easily visible with the lights on and it looked like a toilet. ${ }^{61}$ As mentioned in the behavior section, persons with dementia also seemed to orient strolling behaviors toward areas with increased daylight. ${ }^{52}$ An observational study showed that light level significantly predicted the capacity of residents with dementia to find their way in small-scale group homes, where the light level tended to be lower than in more traditional nursing homes. ${ }^{66}$ These group homes had a larger number of corridors that relied heavily on artificial lighting, Staff knowing their way around and unaware of the importance of light for residents in finding their way around, forgot to switch on the lights and the defunct bulbs made these corridors, even in the middle of the day, very dark.

A long-term intervention with high-intensity neutral light during the day resulted in a significant decrease of cognitive deterioration in the intervention group compared to the control group. ${ }^{43}$ Concerning memory, no significant impact was found of a dawn-dusk simulation. ${ }^{47}$

When investigating engagement while offering stimuli, researchers hypothesized that because of common vision problems persons with dementia would benefit from bright light, and their engagement would be adversely affected by dark surroundings. ${ }^{65}$ Data, however, showed the contrary, quality of engagement (attention and attitude) was significantly more positive in normal light than in bright light. Nonetheless, duration of engagement was significantly more positive when light was normal than in a dark room.

\section{Mood and Emotions}

In all three light intervention studies, depressive symptoms, measured by the Cornell Scale for Depression in Dementia $^{67}$ were significantly improved. ${ }^{43-45}$ However, these light interventions differed considerably in source, color, intensity as well as in duration. A long-term lighting intervention showed a significant decrease in depressive symptoms in the intervention group compared to the control group. ${ }^{43}$ In a before-after study, a 4 -week light intervention significantly decreased depressive symptoms during the intervention period compared to baseline, but the difference lost significance 4 weeks postintervention. ${ }^{45}$ In the third study, the daylight exposure of the intervention group was increased over a period of 12 weeks. ${ }^{44}$ Every morning the participants of the intervention group were taken to a communal room within 3 meters of a daylit window. The control group was taken to a similar sized area indoors under typical electrical lighting conditions without daylight. This resulted in the daylight intervention group showing a significant decrease in depressive symptoms compared to an increase in the control group. Significant group differences were also found for participants with a probable baseline major depression $($ CSDD $>10 ; n=9)$. The intervention subgroup $(n=5)$ had 
a significant reduction in symptoms, comparable to an effective pharmacological treatment.

Five articles reported on the relationship between light and negative affective mood. Two very different light interventions (neutral high-intensity light and extremely cool standard light) yielded no beneficial effects. ${ }^{43,57}$ In a crosssectional study $(\mathrm{n}=160)$ with existing light conditions, persons with dementia who spent many hours in low light levels in their bedrooms showed significant more signs of a negative affective mood, yet no associations were found with light in the living and dining room, where light levels were considerably higher. ${ }^{68}$ As far as negative subjective emotions (anger, sadness and fear) were concerned, no significant differences were found comparing a high light and a low light group. ${ }^{42}$ In a quantitative descriptive study, loneliness and negative emotion had significant negative relationships with satisfaction with indoor light, which in turn was a significant predictor of loneliness. ${ }^{64}$

Positive affective mood was investigated in four very different light conditions and mostly yielded positive results. $^{42,47,64}$ Persons with dementia in a high light group showed significantly more pleasure and higher general alertness than those in a low light group. ${ }^{42}$ However, an intervention with high-intensity neutral light during the day did not produce significant effects on positive affective mood nor on self-esteem in comparison with a control group. ${ }^{43}$ An individually timed dawn-dusk simulation exposure (DDS) for older adults with dementia significantly produced better mood and greater cheerfulness upon awakening in the second 4-week period of the DDS compared to the second 4-week period without DDS. ${ }^{47}$ Only the relatively younger subgroup $(<86$ years; $\mathrm{n}=10$ ) expressed a better mood earlier, namely in the first 4 weeks of the DDS compared to the first 4 weeks without DDS, suggesting that the response may need more time to manifest with higher age. In a quantitative descriptive study, positive emotion, including cheerfulness, was positively predicted by satisfaction with indoor light of persons with dementia. ${ }^{64}$

\section{Quality of Life}

We found results suggesting that daytime light affects the quality of life of persons with dementia in long-term care facilities. Two studies in nursing homes reported a significant positive relationship between light and quality of life of persons with dementia. ${ }^{42,68}$ Measuring the light levels in different rooms (bed room, dining room and living room), the quality of life was positively correlated with the light level of the dining room. ${ }^{68}$ This large crosssectional study $(\mathrm{n}=160)$ found that persons with dementia spending many hours in low light levels in their bedrooms showed more signs of a negative affective mood, which is an element of quality of life. In a between-subjects study $(n=89)$, quality of life was also higher in the high light than in the low light group, but not in the dynamic lighting group when compared to the conventional lighting group. ${ }^{42}$ As mentioned before, this study found no statistical differences in actual light exposure between the dynamic and conventional lighting group. A large crosssectional study ( $\mathrm{n}=194)$ in assisted living facilities using an environmental assessment tool found no significant correlation between quality of life and light intensity, light glare and light evenness. ${ }^{48}$ Finally, DDS had no effect on quality of life either. ${ }^{47}$

\section{Rest-Activity}

The following results concern studies examining the influence of light on rest-activity during the day $(n=8)$, on restactivity at night $(\mathrm{n}=12)$ and on circadian rest-activity rhythms $(n=11)$.

\section{Rest-Activity Daytime}

In general, the light interventions had no effect on daytime rest-activity, apart from the results for some specific subgroups. However, studies in existing light conditions predominantly found a positive correlation between light and activity. Three studies using light interventions of (very) cool light with different light levels during the day showed no significant influence of light exposure on daytime restactivity of persons with dementia. ${ }^{28,42,45}$ However, two of these studies did find significant relationships for specific characteristics, namely severity of dementia and gender. First, in persons with (very) severe dementia, exposure to high-intensity light between 4 and 8 pm was associated with a significant increase in daytime sleepiness. ${ }^{28}$ Second, men in the high light group had significantly higher activity (from 10 am to $8 \mathrm{pm}$ ) than men in the low light group and women in both groups. ${ }^{42}$ A quantitative descriptive study ( $\mathrm{n}=12$ ) in existing light conditions found no relationship between light level and activity during the day. ${ }^{69}$ However, this sample may have been too small to reach significance. In addition, the sample consisted only of women. Two larger cross-sectional studies $\left(n=77 ;^{70}\right.$ $\mathrm{n}=66^{71}$ ) in existing light conditions found a positive relationship between (the peak of) light exposure and (the peak of) activity during the day. ${ }^{70,71}$ One of these studies 
found, "persons with dementia whose peak of light exposure occurred early in the day also had an early peak in activity."71 Thus, for most of these individuals, the peak of light exposure preceded the peak of activity. This study also showed that persons with dementia who spent more time in very high light levels (>2000 lx) had significantly later activity peaks.

\section{Rest-Activity Nighttime}

Most studies ( $n=7 ; 70 \%$; MMAT-score $\geq 3$ ) that examined nighttime rest-activity variables found significant influences of light. ${ }^{42,43,45,64,71-73}$ However, these results were difficult to compare, because of the variety in light conditions and the use of many different - not always clearly defined - sleep variables.

With regard to nocturnal restlessness, no significant influences were found from different light conditions. $^{43,47,69}$ However, a 3-year retrospective longitudinal study did find a specific combination of day length and cloud amount to have an influence on nocturnal restlessness of persons with dementia in nursing homes. ${ }^{73}$ In this study, nighttime activity (indicating nocturnal restlessness) of persons with dementia was significantly higher on cloudy short days than on cloudy long days or on clear short days.

Most studies, including three intervention studies, investigating awakenings at night found no significant influence of light exposure. ${ }^{42,43,47,69,70}$ In contrast, a high quality cross-sectional study in existing light conditions found persons with dementia who had been exposed to higher light levels during the day had significantly fewer awakenings during the night. ${ }^{71}$ Two other studies researched indoor light in a different way. Making efforts to reduce noise and light levels during incontinence care at night, led to a significant decrease in the number of awakenings (related to "light only" and "light and sound") in the intervention group compared to the control group. ${ }^{72}$ Satisfaction with indoor light was another factor associated with less sleep disturbance. ${ }^{64}$

There was no light condition that significantly influenced sleep onset latency. ${ }^{42,43,45,70}$ In a between-subjects study, the high light group showed significant later bedtimes and spent less time in bed than the low light group. ${ }^{42}$ Most of the studies investigating sleep efficiency (including a long-term RCT) ${ }^{43,47,70}$ did not yield any significant results. Only a before-after study showed a significant increase in sleep efficiency as well as in sleep quality during an intervention with very cool light of moderate intensity. ${ }^{45}$ Two light intervention studies, the aforementioned study and an RCT, yielded significant positive results on total sleep time during the night. In this RCT, an intervention with high-intensity neutral light increased total sleep duration by 10 minutes per year for up to 3.5 years. ${ }^{43}$ Studies in existing light conditions and a DDS had no influence on total sleep time during the night. ${ }^{47,69,70}$

\section{4-Hour Rest-Activity Rhythms}

Using the following circadian rest-activity parameters, only a few studies found an influence of light on circadian rest-activity rhythms, such as interdaily stability, intradaily variability, circadian amplitude, 24h-sleep and 24hactivity time. Interdaily stability is used to compare the day-by-day regularity of the 24-hour sleep-wake pattern. ${ }^{45}$ Intradaily variability represents the frequency and extent of transitions between periods of rest and activity during the day. ${ }^{45}$ One repeated measurement study found favorable results for interdaily stability (an increase) and intradaily variability (a decrease) during a light intervention with very high-intensity light, but only when removing persons with severe visual deficiencies from the sample. ${ }^{74}$ Five later published studies exploring different light conditions (not including high-intensity light), yielded no significant results for these two variables, ${ }^{42,45-47,69}$ not even when excluding persons with severe visual deficiencies. ${ }^{42,45}$ One of these studies originally used high-intensity dynamic lighting, but compared circadian rhythms only between the high light and low light group (not being equal to the dynamic and conventional lighting group). ${ }^{42}$

The definitions used for circadian amplitude, amplitude or relative amplitude for the rest-activity cycles are largely, but not entirely, similar. They all represent a difference between the means of the most active 10hour period ("day") and the least active 5-hour period ("night") in the average 24 -hour pattern. ${ }^{75}$ No significant relationships were found between light exposure and amplitude, ${ }^{42,46,47,69,74}$ not even if the studies excluded people with severe eye diseases. ${ }^{42,74}$ The betweensubjects study of Münch et $\mathrm{al}^{42}$ however, has found a gender-specific susceptibility, noting that "higher daily light exposures significantly predicted an increase in relative amplitude only for men." As far as total activity is concerned, this study found no overall greater activity in the high light than in the low light group. In a crosssectional study in existing light conditions, daytime light 
exposure was positively correlated with 24-h activity, but not with $24 \mathrm{~h}$-sleep time. ${ }^{70}$

When other parameters were used to investigate 24hour rest-activity rhythms (phasor magnitude; tympanic temperature), two intervention studies both using very cool light, but of different intensity, found significant evidence for improved circadian rhythms. ${ }^{45,56}$ Nonetheless, an intervention of extremely cool high-intensity light, using tympanic temperature, did not find any notable circadian effects. $^{57}$

\section{Bodily Functions}

Both studies (an $\mathrm{RCT}^{43}$ and a clinical trial ${ }^{76}$ ) showed no increase of adverse effects of the intervention group compared to the control group. Instead, the RCT, a long-term intervention study using high-intensity neutral light significantly lowered the ratings on five items: irritability, dizziness, headache, constipation and inability to sleep. ${ }^{43}$ No significant effects were found on the prescription use of psychotropic medication. ${ }^{43}$ Visual performance was only touched upon in one article. In this study, six caregivers (35.3\%) indicated by questionnaire that the visual performance of their clients improved after installing (dynamic) lighting systems. ${ }^{30}$

\section{Discussion}

This systematic literature review demonstrated that researchers have investigated many different health outcomes in relation to the potential effects of indoor light on older persons with dementia living in long-term care facilities. Behavior was the most researched health outcome, and within this area researchers focused almost exclusively on challenging behaviors. Only two before-after studies in which (very) cool light of moderate intensity was provided showed a significant decrease in agitation. ${ }^{45,46}$ We found no conclusive evidence that periods with less light due to the sunset or to dark seasons caused sundowning behavior. Further, there was no convincing evidence that high-intensity light positively influenced the circadian day-night rhythm, despite a muchcited study from the 1990s showing promising results. ${ }^{74}$ Although many studies showed a beneficial influence of daytime light on nighttime sleep and activity, for each of the variables, it often concerned a single study. In contrast, a 3-year longitudinal study found nocturnal restlessness to be related to a specific combination of day length and cloud amount, namely cloudy short days, ${ }^{73}$ while other light conditions showed no influence. We found that dynamic lighting did not improve day-night rhythm, nor did it improve quality of life..$^{30,42,46}$ However, in one before-after study dynamic lighting decreased agitation. ${ }^{46}$ There were some indications that indoor light exposure during the day positively affected the quality of life of older persons with dementia in long-term care facilities. ${ }^{42,68}$ There are quite strong indications that indoor light can help reduce depressive symptoms and that incident daylight may play an important role in this effect. None of the included studies focused on light for good vision, although light showed to be an important aid for spatial orientation in older persons with dementia. $^{52,61,65,66}$

\section{Non-Seasonal Depression}

Non-seasonal depression is common in older adults with different types of dementia, causing personal (and professional) caregiver distress. ${ }^{77-80}$ Nevertheless, only three studies researched the influence of indoor daylight and lighting on depression in long-term care facilities. These light intervention studies showed promising beneficial effects on depression, sometimes even comparable to an effective pharmacological treatment. ${ }^{43-45}$ An equally small number of research evaluated the treatment of depression in this population using light boxes. ${ }^{81-84}$ The intervention study with the longest treatment period ( 8 weeks) had significant treatment effects on depression, regardless of the severity of dementia. ${ }^{83,84}$ A recent study using aggregated data of three light intervention methods (floor luminaires, light boxes, and light tables) showed a beneficial influence on depressive symptoms as well. ${ }^{85}$ Despite the limited body of research, these combined results confirm the applicability of light to treat and possibly prevent nonseasonal depression among older adults with dementia in long-term care facilities. Probably, indoor daylight and lighting can play an important role in ameliorating depressive symptoms in a more comfortable way than light boxes and with fewer side effects than pharmacological treatment.

\section{Dynamic Lighting}

Imitating natural light indoors was found in the use of dynamic lighting systems. Following up on claims from suppliers, long-term care facilities are installing dynamic lighting systems to improve the well-being and circadian rhythms of older adults with dementia. ${ }^{30}$ Contrary to the industry's compelling claims, research on this topic is still in its early stages and far from conclusive. ${ }^{10}$ The term 
dynamic lighting itself does not provide much clarity. Dynamic lighting scenarios can differ in terms of light intensity, CCT and the timing and duration of both elements. The two dynamic lighting interventions in our review differed in light intensity and CCT, but were similar in duration of light exposure. ${ }^{42,46}$ One of these studies measured the actual daytime light exposure with individual light sensors, and showed that the average daily light exposure did not differ significantly between the group with dynamic lighting and the group with standard lighting. ${ }^{42}$ Despite the fact that both studies were executed in darker seasons, persons in the standard lighting group could go outside or sit in the vicinity of daylit windows, so their light exposure was effectively dynamic too. A review on the rationale for dynamic lighting found several reasons for applying dynamic lighting scenarios, which call for different requirements of the dynamic lighting system. ${ }^{10}$ Using dynamic lighting to entrain the biological clock, for instance, might require another lighting scenario than the ones needed for executing different tasks or activities. Developing effective dynamic lighting scenarios for the different needs of older adults with dementia in long-term care facilities still requires further new high-quality research. For now, the results for this particular study population are inconclusive.

\section{Limitations and Strengths}

This review focused on a clearly defined target group, which enabled us to unravel specific influences of indoor light on older persons with dementia living in long-term care facilities. In addition, we focused on indoor daylight and lighting and excluded studies that (also) focused on other methods of light administration, such as light boxes and light visors, which have different (dis)advantages and characteristics. By combining the results of quantitative, qualitative and mixedmethod studies we were able to include a wide range of research focusing on a broad spectrum of health outcomes. Because of this heterogeneity, however, it was not feasible to undertake a meta-analysis. This heterogeneity also made it difficult to find strong evidence and draw unambiguous conclusions. Additionally, several included studies lacked relevant light data, which hindered comparing and interpreting the results. Finally, though we focused on studies of older adults with dementia, persons with different types of dementia can possibly react differently to light.

\section{Recommendations for Future Research and Practice}

Although ensuring good quality and quantity of light may support older adults with dementia to (more) independently perform everyday activities, only a few of the included studies have focused on this topic. A review on the use of the physical environment to support everyday activities for people with dementia showed overall ADL scores, orientation in space and eating and drinking to be the most frequently researched everyday activities. ${ }^{86}$ The studies included in our review focused precisely on these everyday activities. We recommend further research on the influence and requirements of light to support persons with dementia in performing these and other important everyday activities, such as carrying out hobbies, communicating and getting dressed.

Second, we recommend future research to include accurate descriptions of the light conditions, since almost every study was missing essential elements. To compare and interpret the results, it is imperative that researchers give a clear description of the lighting equipment, the light measurements, the building or room and the exposure to the different light sources, including daylight. ${ }^{10,38}$ Therefore, it is necessary to develop and use consolidated strategies for the description and assessment of both static and dynamic lighting. ${ }^{10}$

Third, future randomized studies are needed to obtain stronger evidence of (very) cool light of moderate intensity to reduce agitation. In addition, further research is needed to clarify the most effective indoor light conditions to prevent or alleviate depressive symptoms. In doing so, we strongly suggest to research the influence of indoor daylight exposure for more than 2 hours per day. We also recommend to clarify which light conditions significantly influence quality of life.

Finally, we recommend both management and care professionals of long-term care facilities to increase their awareness of the potential impact and risks of inadequate light in the daily life of older adults with dementia. Many included articles reported insufficient light conditions in long-term care facilities. A higher quality and quantity of light can support residents to perform everyday activities, to feel better and to prevent unpleasant situations, such as falls and feeling unsafe in dark places. Moreover, this greater quality and quantity of light is also needed for the task performance of (aging) care professionals, for example, to reduce errors in administering medication. ${ }^{87}$ 


\section{Conclusions and Future Directions}

Overall, we found moderate evidence for the influence of indoor light on the health of older persons with dementia living in long-term care facilities. The most promising results of indoor light concerned the reduction of depressive symptoms and the facilitation of spatial orientation. With regard to challenging behaviors, we only found indications for a very specific light intervention to decrease agitation. Further research is needed to corroborate these results in which it is important to describe the characteristics of the light conditions more clearly. No conclusive evidence was found for the influence of indoor light on sundowning behavior, nor on circadian rest-activity rhythms. To date, the research on dynamic lighting has yielded little evidence of its efficacy and has not been sufficiently researched among this study population. Based on our findings, we recommend long-term care facilities to raise awareness of the increased lighting needs of older adults with dementia and the potential beneficial effects of indoor light on health.

\section{Disclosure}

The authors report no conflicts of interest in this work.

\section{References}

1. World Health Organization. The Global Dementia Observatory Reference Guide. Geneva, Switzerland: World Health Organization; 2018.

2. Sloane PD, Mitchell CM, Weisman G, et al. The Therapeutic Environment Screening Survey for Nursing Homes (TESS-NH): an observational instrument for assessing the physical environment of institutional settings for persons with dementia. J Gerontol B Psychol Sci Soc Sci. 2002;57(2):S69-S78. doi:10.1093/geronb/57.2.S69

3. Nolan L. Caring for people with dementia in the acute setting: a study of nurses' views. Br J Nurs. 2007;16(7):419-422. doi:10.12968/ bjon.2007.16.7.23245

4. Day K, Carreon D, Stump C. The therapeutic design of environments for people with dementia: a review of the empirical research Gerontologist. 2000;40(4):397-416. doi:10.1093/geront/40.4.397

5. Van Hoof J, Kort HSM. Supportive living environments: a first concept of a dwelling designed for older adults with dementia. Dementia. 2009;8(2):293-316. doi:10.1177/1471301209103276

6. Van Hoof J, Wouters EJM, Schräder B, et al. Intelligent light therapy for older adults: ambient assisted living. In: Agah A, editor. Medical Applications of Artificial Intelligence. Boca Raton: CRC Press, Taylor \& Francis Group; 2013:343-353.

7. Bouma H, Weale RA, McCreadie C. Technological environments for visual independence in later years. Gerontechnology. 2006;5 (4):187-194. https://journal.gerontechnology.org/archives/641-643-1PB.pdf

8. Van Someren EJW, Hagebeuk EEO, Lijzenga C, et al. Circadian rest -activity rhythm disturbances in alzheimer's disease. Biol Psychiatry. 1996;40(4):259-270. doi:10.1016/0006-3223(95)00370-3

9. Aries MBC, Vlies RD, Westerlaken AC. Inventarisatie en vastlegging van de State-of-Art kennis over licht en ouderen. Utrecht: TNO; 2010 .

10. Kompier ME, Smolders KCHJ, de Kort YAW. A systematic literature review on the rationale for and effects of dynamic light scenarios. Build Environ. 2020;186:1-12. doi:10.1016/j.buildenv.2020.107326
11. Wever RA, Polášek J, Wildgruber CM. Bright light affects human circadian rhythms. Pflugers Arch. 1983;396(1):85-87. doi:10.1007/ BF00584704

12. Campbell SS, Dijk D-J, Boulos Z, Eastman CI, Lewy AJ, Terman M. Light treatment for sleep disorders: consensus report: III. Alerting and activating effects. $J$ Biol Rhythms. 1995;10(2):129-132. doi:10.1177/074873049501000205

13. Lockley SW. Circadian rhythms: influence of light in humans. In: Squire LR, editor. Encyclopedia of Neuroscience. 5th ed. Oxford, England: Academic Press; 2009:971-986.

14. Turner PL, Van Someren EJW, Mainster MA. The role of environmental light in sleep and health: effects of ocular aging and cataract surgery. Sleep Med Rev. 2010;14(4):269-280. doi:10.1016/j. smrv.2009.11.002

15. Van Someren EJW, Riemersma-van Der Lek RF. Live to the rhythm, slave to the rhythm. Sleep Med Rev. 2007;11(6):465-484. doi:10.1016/j.smrv.2007.07.003

16. De Lepeleire J, Bouwen A, De Coninck L, Buntinx F. Insufficient lighting in nursing homes. J Am Med Dir Assoc. 2007;8(5):314-317. doi:10.1016/j.jamda.2007.01.003

17. Sinoo MM, Van Hoof J, Kort HSM. Light conditions for older adults in the nursing home: assessment of environmental illuminances and colour temperature. Build Environ. 2011;46(10):1917-1927. doi:10.1016/j.buildenv.2011.03.013

18. Sloane PD, Mitchell CM, Calkins M, Zimmerman SI. Light and Noise Levels in Alzheimer's Disease Special Care Units. Vol. 4. New York: Springer; 2000.

19. Moore KJ, Hill KD, Robinson AL, Haines TP, Haralambous B, Nitz JC. The state of physical environments in Australian residential aged care facilities. Aust Health Rev. 2011;35(4):412-417. doi:10.1071/AH10932

20. Figueiro MG, Hamner R, Higgins P, Hornick T, Rea MS. Field measurements of light exposures and circadian disruption in two populations of older adults. J Alzheimers Dis. 2012;31(4):711-715. doi:10.3233/JAD-2012-120484

21. McCurry SM, Reynolds CF, Ancoli-Israel S, Teri L, Vitiello MV. Treatment of sleep disturbance in Alzheimer's disease. Sleep Med Rev. 2000;4(6):603-628. doi:10.1053/smrv.2000.0127

22. Roccaro I, Smirni D. Fiat lux: the light became therapy. An overview on the bright light therapy in Alzheimer's disease sleep disorders. J Alzheimers Dis. 2020;77(1):113-125. doi:10.3233/JAD-200478

23. Guo L, Duggan J, Cordeiro MF. Alzheimer's disease and retinal neurodegeneration. Curr Alzheimer Res. 2010;7(1):3-14. doi:10.2174/156720510790274491

24. Van Hoof J, Aarts MPJ, Westerlaken AC, et al. Light therapy in smart healthcare facilities for older adults: an overview. In: Curran K, editor. Recent Advances in Ambient Intelligence and Context-Aware Computing. Hershey, PA, USA: IGI Global; 2015:303-310.

25. Konis K. Field evaluation of the circadian stimulus potential of daylit and non-daylit spaces in dementia care facilities. Build Environ. 2018;135:112-123. doi:10.1016/j.buildenv.2018.03.007

26. Colenda CC, Cohen W, McCall WV, Rosenquist PB. Phototherapy for patients with Alzheimer disease with disturbed sleep patterns: results of a community-based pilot study. Alzheimer Dis Assoc Disord. 1997;11(3):175-178. doi:10.1097/00002093-19970900000011

27. Barrick AL, Sloane PD, Williams CS, et al. Impact of ambient bright light on agitation in dementia. Int $J$ Geriatr Psychiatry. 2010;25 (10):1013-1021. doi:10.1002/gps.2453

28. Sloane PD, Williams CS, Mitchell CM, et al. High-intensity environmental light in dementia: effect on sleep and activity. $\mathrm{J} \mathrm{Am}$ Geriatr Soc. 2007;55(10):1524-1533. doi:10.1111/j.15325415.2007.01358.x

29. Izsó L, Laufer L, Suplicz S. Effects of dynamic lighting on the visual performance of older adults. Light Res Technol. 2009;41:361-370. doi: $10.1177 / 1477153509336802$ 
30. Aarts MPJ, Aries MBC, Straathof J, Van Hoof J. Dynamic lighting systems in psychogeriatric care facilities in the Netherlands: a quantitative and qualitative analysis of stakeholders' responses and applied technology. Indoor Built Environ. 2015;24(5):617-630. doi:10.1177/1420326X14532387

31. Forbes D, Blake CM, Thiessen EJ, Peacock S, Hawranik P. Light therapy for improving cognition, activities of daily living, sleep, challenging behaviour, and psychiatric disturbances in dementia. Cochrane Database Syst Rev. 2014;(2). doi:10.1002/14651858. CD003946.pub4.

32. Missotten P, Farag L, Delye S, Muller A, Grotz C, Adam S. Role of "light therapy" among older adults with dementia: an overview and future perspectives. Geriatr Psychol Neuropsychiatr Vieil. 2019;17 (1):83-91. doi:10.1684/pnv.2019.0786

33. Aries MBC, Aarts MPJ, Van Hoof J. Daylight and health: a review of the evidence and consequences for the built environment. Light Res Technol. 2015;47(1):6-27. doi:10.1177/1477153513509258

34. Shikder S, Mourshed M, Price A. Therapeutic lighting design for the elderly: a review. Perspect Public Health. 2012;132(6):282-291. doi: $10.1177 / 1757913911422288$

35. Huber M, Knottnerus JA, Green L, et al. How should we define health? Br Med J. 2011;343:d4163-d4163. doi:10.1136/bmj.d4163

36. World Health Organization. Constitution of the World Health Organzation; 2006. Available from: www.who.int/governance/eb/. Accessed April 21, 2021.

37. Moher D, Liberati A, Tetzlaff J, Altman DG. The PRISMA Group. Preferred Reporting Items for Systematic Reviews and MetaAnalyses: The PRISMA Statement. PLoS Med. 2009;6(7):e1000097. doi:10.1371/journal.pmed.1000097

38. Aarts MPJ, Aries MBC, Diakoumis A, Van Hoof J. Shedding a light on phototherapy studies with people having dementia: a critical review of the methodology from a light perspective. $\mathrm{Am}$ J Alzheimers Dis Other Demen. 2016;31(7):551-563. doi:10.1177/ 1533317515628046

39. Hong QN, Pluye P, Fàbregues S, et al. Mixed methods appraisal tool (MMAT), version 2018. Registr Copyright. 2018;1148552.

40. Rheaume YL, Manning BC, Harper DG, Volicer L. Effect of light therapy upon disturbed behaviors in Alzheimer patients. Am J Alzheimers Dis Other Demen. 1998;13(6):291-295.

41. Cohen-Mansfield J, Marx MS, Rosentahl AS. A description of agitation in a nursing home. $J$ Gerontol. 1989;44(3):M77-M84. doi:10.1093/geronj/44.3.M77

42. Münch M, Schmieder M, Bieler K, et al. Bright light delights: effects of daily light exposure on emotions, rest-activity cycles, sleep and melatonin secretion in severely demented patients. Curr Alzheimer Res. 2017;14(10):1063-1075. doi:10.2174/1567205014666170523 092858

43. Riemersma-van der Lek RF, Swaab DF, Twisk J, et al. Effect of bright light and melatonin on cognitive and noncognitive function in elderly residents of group care facilities: a randomized controlled trial. JAMA. 2008;299(22):2642-2655. doi:10.1001/jama.299. 22.2642

44. Konis K, Mack WJ, Schneider EL. Pilot study to examine the effects of indoor daylight exposure on depression and other neuropsychiatric symptoms in people living with dementia in long-term care communities. Clin Interv Aging. 2018;13:1071-1077. doi:10.2147/ CIA.S165224

45. Figueiro MG, Plitnick BA, Lok A, et al. Tailored lighting intervention improves measures of sleep, depression, and agitation in persons with Alzheimer's disease and related dementia living in long-term care facilities. Clin Interv Aging. 2014;9:1527-1537. doi:10.2147/ CIA.S68557

46. Wahnschaffe A, Nowozin C, Haedel S, et al. Implementation of dynamic lighting in a nursing home: impact on agitation but not on rest-activity patterns. Curr Alzheimer Res. 2017;14(10):1076-1083. doi: $10.2174 / 1567205014666170608092411$
47. Bromundt V, Wirz-Justice A, Boutellier M, et al. Effects of a dawn-dusk simulation on circadian rest-activity cycles, sleep, mood and well-being in dementia patients. Exp Gerontol. 2019;24 (110641):1-8. doi:10.1016/j.exger.2019.110641

48. Bicket MC, Samus QM, McNabney M, et al. The physical environment influences neuropsychiatric symptoms and other outcomes in assisted living residents. Int $J$ Geriatr Psychiatry. 2010;25 (10):1044-1054. doi:10.1002/gps.2460

49. Cohen-Mansfield J, Thein K, Marx MS, Dakheel-Ali M, Murad H, Freedman LS. The relationships of environment and personal characteristics to agitated behaviors in nursing home residents with dementia. J Clin Psychiatry. 2012;73(3):392-399. doi:10.4088/ JCP. $10 \mathrm{~m} 06605$

50. Elmståhl S, Annerstedt L, Ahlund O. How should a group living unit for demented elderly be designed to decrease psychiatric symptoms? Alzheimer Dis Assoc Disord. 1997;11(1):47-52. doi:10.1097/ 00002093-199703000-00008

51. Martin J, Marler M, Shochat T, Ancoli-Israel S. Circadian rhythms of agitation in institutionalized patients with Alzheimer's disease. Chronobiol Int. 2000;17(3):405-418. doi:10.1081/CBI-100101054

52. Mobley C, Leigh K, Malinin L. Examining relationships between physical environments and behaviors of residents with dementia in a retrofit special care unit. J Inter Des. 2017;42(2):49-69. doi:10.1111/joid.12094

53. Algase DL, Beattie ERA, Antonakos C, Beel-Bates CA, Lan Y. Wandering and the physical environment. Am J Alzheimers Dis Other Demen. 2010;25(4):340-346. doi:10.1177/1533317510365342

54. Cohen-Mansfield J, Werner P, Marx MS, Freedman L. 2 studies of pacing in the nursing home. J Gerontol. 1991;46(3):M77-M83. doi:10.1093/geronj/46.3.M77

55. Jao YL, Algase DL, Specht JK, Williams K. The association between characteristics of care environments and apathy in residents with dementia in long-term care facilities. Gerontologist. 2015;55(Suppl 1):S27-39. doi:10.1093/geront/gnu166

56. Van Hoof J, Aarts MPJ, Rense CG, Schoutens AMC. Ambient bright light in dementia: effects on behaviour and circadian rhythmicity. Build Environ. 2009;44(1):146-155. doi:10.1016/j.buildenv.20 08.02.005

57. Van Hoof J, Schoutens AMC, Aarts MPJ. High colour temperature lighting for institutionalised older people with dementia. Build Environ. 2009;44(9):1959-1969. doi:10.1016/j.buildenv.2009.01.009

58. Sloane PD, Mitchell CM, Preisser JS, Phillips C, Commander C, Burker E. Environmental correlates of resident agitation in Alzheimer's disease special care units. J A Geriatr Soc. 1998;46 (7):862-869. doi:10.1111/j.1532-5415.1998.tb02720.x

59. Wong JKW, Skitmore M, Buys L, Wang K. The effects of the indoor environment of residential care homes on dementia suffers in Hong Kong: a critical incident technique approach. Build Environ. 2014;73:32-39. doi:10.1016/j.buildenv.2013.12.001

60. Bliwise DL, Carroll JS, Lee KA, Nekich JC, Dement WC. Sleep and "sundowning" in nursing home patients with dementia. Psychiatry Res. 1993;48(3):277-292. doi:10.1016/0165-1781(93)90078-U

61. Coulson I, White J. A total environment quality of care approach to evaluation of management and care at two dementia care units in Tasmania. Am J Alzheimers Dis Other Demen. 1997;12 (3):128-137. doi:10.1177/153331759701200306

62. Chang CC, Lin YF, Chiu CH, et al. Prevalence and factors associated with food intake difficulties among residents with dementia. PLoS One. 2017;12(2):e0171770. doi:10.1371/journal.pone.0171770

63. Brush JA, Meehan RA, Calkins MP. Using the environment to improve intake for people with dementia. Alzheimers Care $Q$. 2002;3(4):330-338.

64. Leung MY, Wang C, Wei X. Structural model for the relationships between indoor built environment and behaviors of residents with dementia in care and attention homes. Build Environ. 2020;169:106532. doi:10.1016/j.buildenv.2019.106532 
65. Cohen-Mansfield J, Thein K, Dakheel-Ali M, Marx MS. Engaging nursing home residents with dementia in activities: the effects of modeling, presentation order, time of day, and setting characteristics. Aging Ment Health. 2010;14(4):471-480. doi:10.1080/13607860903586102

66. Netten A. The effect of design of residential homes in creating dependency among confused elderly residents: a study of elderly demented residents and their ability to find their way around homes for the elderly. Int $J$ Geriatr Psychiatry. 1989;4(3):143-153. doi:10.1002/gps.930040305

67. Alexopoulos GS, Abrams RC, Young RC, Shamoian CA. Cornell scale for depression in dementia. Biol Psychiatry. 1988;23 (3):271-284. doi:10.1016/0006-3223(88)90038-8

68. Garre-Olmo J, López-Pousa S, Turon-Estrada A, Juvinyà D, Ballester D, Vilalta-Franch J. Environmental determinants of quality of life in nursing home residents with severe dementia. J Am Geriatr Soc. 2012;60(7):1230-1236. doi:10.1111/j.1532-5415.2012.04040.x

69. Song Y, Dowling GA, Wallhagen MI, Lee KA, Strawbridge WJ, Hubbard EM. Rest-activity patterns in institutionalized Korean older adults with dementia: a pilot study. J Gerontol Nurs. 2009;35 (12):20-30. doi:10.3928/00989134-20091109-99

70. Ho J, Mathews RM, Heard R, Chin Moi C. Differences in sleep of dementia residents between Macao (China) and Sydney (Australia). Macau J Nurs. 2013;12(2):52.

71. Shochat T, Martin J, Marler M, Ancoli-Israel S. Illumination levels in nursing home patients: effects on sleep and activity rhythms. J Sleep Res. 2000;9(4):373-379. doi:10.1046/j.1365-2869.2000.00221.x

72. Schnelle JF, Alessi CA, Al-Samarrai NR, Fricker RD, Ouslander JG. The nursing home at night: effects of an intervention on noise, light, and sleep. J Am Geriatr Soc. 1999;47(4):430-438. doi:10.1111/ j.1532-5415.1999.tb07235.x

73. Wahnschaffe A, Nowozin C, Rath A, et al. Night-time activity forecast by season and weather in a longitudinal design - natural light effects on three years' rest-activity cycles in nursing home residents with dementia. Int Psychogeriatr. 2017;1-10. doi:10.1017/ S1041610217001235

74. Van Someren EJW, Kessler A, Mirmiran M, Swaab DF. Indirect bright light improves circadian rest-activity rhythm disturbances in demented patients. Biol Psychiatry. 1997;41(9):955-963. doi:10.1016/S0006-3223(97)89928-3

75. Witting W, Kwa IH, Eikelenboom P, Mirmiran M, Swaab DF. Alterations in the circadian rest-activity rhythm in aging and Alzheimer's disease. Biol Psychiatry. 1990;27:536-572. doi:10.1016/0006-3223(90)90523-5

76. Sloane PD, Noell-Waggoner E, Hickman S, et al. Implementing a lighting intervention in public areas of long-term care facilities: lessons learned. Alzheimers Care Q. 2005;6(4):280-293.
77. Andreasen P, Lönnroos E, von Euler-chelpin MC. Prevalence of depression among older adults with dementia living in low-and middle-income countries: a cross-sectional study. Eur J Public Health. 2014;24(1):40-44. doi:10.1093/eurpub/ckt014

78. Enache D, Winblad B, Aarsland D. Depression in dementia: epidemiology, mechanisms, and treatment. Curr Opin Psychiatry. 2011;24 (6):461-472. doi:10.1097/YCO.0b013e32834bb9d4

79. Steinberg M, Shao H, Zandi P, et al. Point and 5-year period prevalence of neuropsychiatric symptoms in dementia: the Cache County Study. Int J Geriatr Psychiatry. 2008;23(2):170-177. doi:10.1002/gps. 1858

80. Tan LL, Wong HB, Allen H. The impact of neuropsychiatric symptoms of dementia on distress in family and professional caregivers in Singapore. Int Psychogeriatr. 2005;17(2):253. doi:10.1017/ S1041610205001523

81. Burns A, Allen H, Tomenson B, Duignan D, Byrne J. Bright light therapy for agitation in dementia: a randomized controlled trial. Int Psychogeriatr. 2009;21(4):711. doi:10.1017/S104161020900 8886

82. Lyketsos CG, Lindell Veiel L, Baker A, Steele C. A randomized, controlled trial of bright light therapy for agitated behaviors in dementia patients residing in long-term care. Int $J$ Geriatr Psychiatry. 1999;14(7):520-525. doi:10.1002/(SICI)1099-1166(199907)14:7<520::AID-GPS983>3.0.CO;2-M

83. Onega LL, Pierce TW, Epperly L. Effect of bright light exposure on depression and agitation in older adults with dementia. Issues Ment Health Nurs. 2016;37(9):660-667. doi:10.1080/01612840. 2016.1183736

84. Onega LL, Pierce TW, Epperly L. Bright light therapy to treat depression in individuals with mild/moderate or severe dementia. Issues Ment Health Nurs. 2018;39(5):370-373. doi:10.1080/ 01612840.2018 .1437648

85. Figueiro MG, Plitnick B, Roohan C, Sahin L, Kalsher M, Rea MS. Effects of a tailored lighting intervention on sleep quality, restactivity, mood, and behavior in older adults with Alzheimer disease and related dementias: a randomized clinical trial. J Clin Sleep Med. 2019;15(12):1757-1767. doi:10.5664/jcsm.8078

86. Woodbridge R, Sullivan MP, Harding E, et al. Use of the physical environment to support everyday activities for people with dementia: a systematic review. Dementia (London). 2018;17(5):533-572. doi: $10.1177 / 1471301216648670$

87. Aarts MPJ, Craenmehr G, Rosemann ALP, Van Loenen EJ, Kort HSM. Light for patient safety: impact of light on reading errors of medication labels. Int J Ind Ergon. 2019;71:145-154. doi:10.1016/ j.ergon.2019.03.004
Clinical Interventions in Aging

\section{Publish your work in this journal}

Clinical Interventions in Aging is an international, peer-reviewed journal focusing on evidence-based reports on the value or lack thereof of treatments intended to prevent or delay the onset of maladaptive correlates of aging in human beings. This journal is indexed on PubMed Central, MedLine, CAS, Scopus and the Elsevier
Bibliographic databases. The manuscript management system is completely online and includes a very quick and fair peer-review system, which is all easy to use. Visit http://www.dovepress.com/ testimonials.php to read real quotes from published authors. 